PPPL--2222

DE85 013761

\title{
Current in Wave Driven Plasmas
}

\author{
Charles F. F. Karney and Nathaniel J. Fisch \\ Plasma Physics Laboratory, Princeton University \\ Princeton, NJ 08544
}

\begin{abstract}
A theory for the generation of current in a toroidal plasma by radio-frequency waves is presented. The effect of an opposing electric field is included, allowing the case of time varying currents to be studied. The key quastities tbat character's : this regime are identified and aumerically calculated. Circuit equations suitable for use in ray-tracing and transport codes are giver.
\end{abstract}

\section{DISCLAMMER}

an acount of work sponsored by an agency of the United States This repart was prepared as an acoount of wotk apons nor any agency thereof, not any of their Government. Neither the United Stated Gaverament nor any agency legal libbility or responsiemployees, riakes any warranty, express or implied, or assunes any log apparatus, product, or bility for the aceuracy, completeness, of usefulness of any information, apparad rights. Refermuess disclased, or represents that its use would not infrioge five by trade name, trademark. ence berein to any specific commercial produat, process, or servity is endorsemeat, recom. manufacturer, or otherwise does not necessarily constion or any ageney thereot. The views mendation, or favoring by the United States Governwessarily state or reflect those of the and opinions of authors expressed herein do agl

United States Govemment or any agency thereof. 


\section{INTRODUCTION}

In recent years there has been congiderable interest in generating steadystate currents in a plasma with if wayes. In particular, it was predicted' that these currents could be efficiently gegerated by waves whose phase velocities are several times the electron thermal speed. This prediction bas been confirmed by numerous experiments in which the current was driven by lower-bybrid waves. These results allow us to contemplate a steady-state tokamak reactor in which the toroidal current is driven by lower-hybrid waves. This is an attractive proposition not only because of the advantages inherent in steady-state operation (less thermal stress, higher duty cycle, etc.), but also becauae it opens up the possibility that the ohmic winding of the tokanal can be eliminated entireiy, leading to a cheaper and more compact reactor. This latter possibility can be realized if if waves are succeseful not only in sugtaining the plasma current "steady-state current drive," but also in increasing the plasma current "If current ramp-up." In fact, experiments have demonstrated that this too is possible. From a tieoretical point of view, the important additional ingredient in these experiments is the de electric field which opposes the increase of the plasma current. The electric field is also present in schemes where the of is used to recbarge the trangformer at conotant curreat.

Recently, we presented a theory for of current drive in the presence of an electric field. ${ }^{2}$ This theory predicted that rf energy could be efficiently converted to poloidal field energy if the wave phase velocity were approximately equal to the electron runaway velocity. This sheory has been compareds witb data from the PLT experiment, 4 and excellent agreement is found. In Ref. 2, the linearized Boltzmann equation was approximateły solved by integrating the corresponding Langevin equations using a Monte-Carlo method. In this paper, we use a more elegant theory to calculate the efficiency of the current ramp-up based on an adjoint formulation for the Boltzmann equation. ${ }^{5}$ Aithcugh the limits of validity of this theory are the same as for Ref. 2, this theory is more amenable to accurate evaluation on a computer, and it is more easily extended to include effects which are omitted here.

Let us begin by reiterating the physical picture given in Ref. 2. Consider an electron travelling in the positive direction at several times the thermal speed and which has just absorbed an incremental amount of if energy. Suppose there is an electric field tending to decelesate this electron. The question is: Where does thin incremental enersy end up? If the electron is siow compared to the runaway velocity, the electron slows down primarily due to collisions and so the if energy goes to bulk heating. On the other hand, if the electron ws fast, the electron is slowed down by the electric field. In this case, the if energy is coupled to the plasma circuit and appeans as poloidal field energy. Unfortnnately, fast electrons bave a high probability of pitch angle scattering into the reveree direction and nuning away. A rubaway electron drains energy 
out of the electric feld, leading to a degradatic of the ramp-up efficiency. However, there is a window around the runaway volocity where the ulectroas are slowed down principally by the electric field and yet where the probability of runping away is very small. This is the favorable regime in which if energy can be efficientiy converted to polvidal field eneroy.

From the foregoing discussion, we see that two ingredieats are needed for an accurate theoretical treatment of this problem. First, the electric field must. be treated an large. In the efficient regime, tive force on the electron due to the electric field must be comparable to that due to collisions. Second, a two-dimensional treatment is required. Analyses based on a ont-dimensional Fokker-Planck equation do not predict the iraportant physical phenomenon of rf-generated reverse runaways.

We briedy review the history of theoretical studies of current drive in the presente of an electric fiejd. The earliest theoretical studies ${ }^{1}$ of lower-bybrid current drive assumed that there was no electric field in the plasme. This is the appropriate limit for a steady-state reactor. However, some of the early experiments eonducted to verify the predictions of the theory tvere conducted in regimes where the ohmic electric beld was still present. This prompted a series of papers ${ }^{0,7,8,9,10}$ dealing with if current drive in the presence of an assisting electric field (i.e., the electric field and the if botb aceelerate the electrons in the same direction). The principal focus of these papers was the calculation of an en hanced runaway rate when the phase velocity of the waves is in the neighborhood of the runaway velocity. An opposing electric feld was treated by Borrass and Nocentini" "within tte framework of a one-dimensiona 1 Fokker-Planck analyais. A similar model has been employed mare recently by Liu et al ${ }^{12}$ As noted above, snch an analysis cannot include if-generated reverse runaways. Also due to the crudity of the one-dimensional equation, the results are only accurate to within a factor of order unity even when ac runaways are geoerated. A two dimensional treatment of the problem in the small electric field limit has been given by Start ${ }^{13}$ for the case of eurrent drive by electron-cyclotron waves. This work negiected election-electron collision. This defect was removed and the results generalized to arbitrary current-drive methods by Fisch ${ }^{14}$ employing an adjoint formulation. This wort yields acearate results when the electric field is small. However, the results are inapplicable in the regime of efficient ramp-up where the phase velocity is comparable to the rusaway velocity. Our earlier paper $^{2}$ was the first tw combine a two-dimensional treatment with a large electric feld. This paper allowed an aceurate calculation of the ramp-up efficiency in cases of prectical interest, ard identifed the regime in which high efficieneies can be expected. The present work is a continuation and expansion of that earlier paptr. Besides using the more sophisticated method ${ }^{5}$ for solving the Boltamann equation, we endeavor to give the results in a form that allows both easy comparison with experiments agd easy implementation within the framework of ray-tracing or trawsport codes. 
The paper is organized as follows: We begin with the linearized Boltzmarn equation for the perturbed electron distribution in the presence of an electric field and an if source (Sec. II). Some approximatioso and normalizations are made to reduce this equation to a more manageable form. The use of the adjoint methods for solving the reoulting equation is described (Sec. III). Next (Sec. IV) the adjoint equation is solved numerically to give the runawray probability and the Green's function for the current. The latter quantity is reduced to a simple form which involves just two functions of velocity. An expression for the total current density is given (Sec. V) and this is put into a form which is easy to calculate. How the If-driven current interacts with the electric field to produce poloidal field energy is considered (Sec. Vi), and the results are applied to experiments.

\section{BOLTZMANN BQUATION}

Consider a uniform electron-ion plasma, initially at equilibrium. For $t>0$, it is subject to an electric field $\mathbf{E}(t)$ and a wave-induced llux $S(v, t)$. We will take the jons to be infinitely massive, so that they form a stationary backgroind of which the eleetrons collide. If the electric field and the wave-induced flux are wesk enougb, the electron dirtribution remains close to a Maxwellian for $\varepsilon \leqslant T$ where $\varepsilon$ is the exergy of an electron $\frac{1}{2} m v^{2}$. Substituting $f=f_{m}+f_{1}$ into the Boltzmann equation for the elettron distribution $f$ and linearizing then gives

$$
\begin{aligned}
\frac{\partial}{\partial t} f_{1}+\frac{q \mathbf{E}(t)}{m} \cdot \frac{\partial}{\partial v} f_{1}-C\left(f_{1}\right\} & \\
& =-\frac{\partial}{\partial v} \cdot \mathbf{S}-\frac{q \mathbf{E}(t)}{m} \cdot \frac{\partial}{\partial v} f_{m}-\left[\frac{\dot{n}}{n}+\left(\frac{\varepsilon}{T}-\frac{3}{2}\right) \frac{\dot{T}}{T}\right] f_{m},
\end{aligned}
$$

where

$$
f_{m}=n\left(\frac{m}{\delta \pi T}\right)^{3 / 2} \exp (-\varepsilon / T)
$$

and

$$
C(f)=C\left(f, f_{m}\right)+C\left(f_{m}, f\right)+C\left(f, f_{i}\right)
$$

is the linearized collision operator. Here $q, m, n$, and $T$ are the electron charge, mass, number density, and temperature. Note that $q$ carries the sign of the electron charge (i.e., $q=-6$ ).

This equation is to be solved with initial condition $f_{I}(v, t=0)=0$. We demand that the subsequeit evolution of $f_{1}$ be such that it be orthogonal to 1 and $\mathcal{E}$, i.e., that it have zero denuity and energs: The zero-density condition is gatisfied with $\dot{n}=0$ since all the terms in $\mathrm{Eq}$. (1) are particle conserving. The zero-energy condition gives an equation for the time evolution of $T$

$$
\frac{3}{2} n \frac{d T}{d t}=\int \mathrm{mS} \cdot \mathbf{v} d^{2} \mathbf{v}+\mathbf{E} \cdot \int q v f_{1} d^{3} \mathbf{v}
$$


The two tertas on the right tand side represent the beatiog due to the waves and due to $\mathbf{E} \cdot \mathbf{J}$.

We now make three simplifying assumptions: we avgume that $f_{1}$ is azimuthally symmetric about the ambient magnetic field; we take the electric feld to be constant and in the direction parallel to the magnetic field $\mathbf{E}=E v_{\text {il }}$; and we restrict our aftention to those cases where $\mathbf{S}$ is only finite where $v \rightarrow v_{t}$, where $v_{t}^{2}=T / m$ is the thermal velocity. We may then solve Eq. (1) using the bigh-velocity form for $C$ :

$$
C(f)=\Gamma\left[\frac{1}{v^{2}} \frac{\partial}{\partial v} f+\frac{1+Z}{2 v^{3}} \frac{\partial}{\partial \mu}\left(1-\mu^{2}\right) \frac{\partial}{\partial \mu} f\right],
$$

where $\mu=v_{\|} / v, \Gamma \doteq m q^{4} \ln L / 4 \pi \epsilon_{0}^{2} m^{2}$, to is the dielectric constant of free space, In $A$ is the Coulomb logarithm, and $Z$ is the effective ion charge state. We have included pitch-angle scattering and fricticnal slowing down, but ignored energy diffusion. If the problem of steady-state current drive, ${ }_{1}^{13}$ the energy difission term introduces corrections of order $\left(v_{t} / v\right)^{2}$. Another term neglected in this approximate collision operator is the effect of the Maxwellian colliding off the perturbed distribution, $C\left(f_{m}, f\right)$. The corrections due to this term ${ }^{15}$ are of order $\left(v_{t} / v\right)^{3}$. With these approximations, the colligion operator does not depend on the electron temperature $T$. Formally, we may derive the form for $C$ by taking $T \rightarrow 0$. In this limit, we have $f_{\mathrm{m}} \rightarrow \mathrm{n} \delta(\mathrm{v})$.

It is convenient to introduce some normalizatione. The runaway velocity $v$, is that velocity at which collisional frictional force equals the acceleration due to the electric field

$$
v_{r} \equiv-\operatorname{sign}(q E) \sqrt{m \Gamma /|q E|} .
$$

Notice that the sign of $v_{\tau}$ is oppcsite to the direction in which electrons run away. The Dreicer velocity ${ }^{10}$ is given by $-\sqrt{2+Z} v_{r}$. Similarly, we define a runaway collision frequency

$$
\nu_{\mathrm{r}} \equiv \boldsymbol{\Gamma} /\left|v_{\mathrm{r}}\right|^{3} \text {. }
$$

The normalized time and velacity are given by

$$
r=u_{r} t
$$

and

$$
\mathbf{u}=\mathbf{v} / \mathrm{v}_{\mathbf{r}} .
$$

The components of $\mathbf{v}$ have to be normalized with care: $u_{\perp}=v_{\perp} /\left|v_{r}\right|_{,} u_{\|}=$ $v_{\|} / v_{r}$, and $u=v /\left|v_{r}\right|$. This implies that $u_{\|} / u=\operatorname{sign}\left(v_{r}\right) v_{\|} / v$ so that the conversion of the pitch-angle variable $\mu$ in $\mathbf{v}$ space to that in $u$ space involves multiplication by sigo $\left(v_{r}\right)$. Other quantities are normalized in a similar way: however, we shall use the same symbols as tor the unnormalized quantities. Thus, the distribution functions $f_{1}$ and $f_{m}$ are normalized to $n /\left|v_{r}\right|^{3}$, the rfinduced lux $S$ to $n \nu_{r} v_{r} /\left|v_{r}\right|^{3}$, etc. Under this normalization, Eq. (1) then 
becomes

$$
\frac{\partial}{\partial \tau} f_{1}+D\left(f_{1}\right)=-\frac{\partial}{\partial u} \cdot \mathbf{S},
$$

with $f_{1}(u, \tau=0)=0$ and with the operator $D$ defined by

$$
D \equiv-\frac{\partial}{\partial u_{\|}}-\frac{1}{u^{2}} \frac{\partial}{\partial u}-\frac{1+Z}{2 u^{3}} \frac{\partial}{\partial \mu}\left(1-\mu^{2}\right) \frac{\partial}{\partial \mu} .
$$

Equation (2) is singular at the origin. However, becauge $f_{1}$ in Eq. (2) describes a physical particle distribution, it obeys a particle conservation law near the origin. We therefore require that, close to $u=0, f_{1}(\mathbf{u})=N(r) \delta(u)$ with $N(0)=0$ and

$$
\frac{d N}{d \tau}=\lim _{x \rightarrow 0} \int_{-t}^{1} 2 \pi f_{1}(u, \mu) d \mu .
$$

Equation (2) depends only on a single parameter $Z$. The dependence on the electric feld $E$ can be normalized away, since the electric field defines the only natural velocity scale in the problem, $v_{r}$.

Equation (2) is amenable to various method of solution, and it is instructive to review these before describing the method used here. The most straightforward approach is to integrate $\mathrm{Eq}$. (2) directly or: a computer. This method allows $\mathbf{S}$ to be determined directly in terms of the electron distribution $f$. However, a thorough understanding of the problem requires that many different forms of $\mathbf{S}$ be used. Therefore, this procedure is costly because the several parameters used to specify $\mathbf{S}$ must be scanned. This is essentially the method adopted in the early numerical studies of steady-state current drive by lewetbybrid waves. ${ }^{17}$

The situation is improved to some ertent by noting that $\mathrm{Eq} .(2)$ is a linear equation for $f_{1}$. It may be solved in terms of a Green's function g given by the equation

$$
\left(\frac{\partial}{\partial r}+D\right) g\left(u, \tau ; u^{\prime}\right)=0
$$

with $g\left(\mathbf{u}, r=0 ; \mathbf{u}^{\prime}\right)=\delta\left(\mathbf{u}-\mathbf{u}^{\prime}\right)$. The electron distribution is then given by $t$ he convolution

$$
f_{1}(u, \tau)=\int_{0}^{\tau} d r \int d^{\prime} u^{\prime} \mathbf{S}\left(\mathbf{u}^{\prime}, \tau^{\prime}\right) \cdot \frac{\partial}{\partial \mathbf{u}^{\prime}} g\left(\mathbf{u}, \tau-\tau^{\prime} ; \mathbf{u}^{\prime}\right)
$$

This approach reduces the problem to the determination of a single function $g$ of two vector arguments $\left(u\right.$ and $\left.\mathbf{u}^{\prime}\right)$ and a one scalar argument $\{r\}$. However this is otill a daunting computational task.

A closely related technique is to formulate the problem as a set of Langevin equations, ${ }^{10}$

$$
\begin{aligned}
& \frac{d u}{d t}:=-\frac{1}{u^{2}}-\mu_{1} \\
& \frac{d \mu}{d \tau}=A(\tau)-\frac{1-\mu^{2}}{u},
\end{aligned}
$$


where the pitch-angle scattering is represcated by the stochastic term $A(\tau)$. Assuming that $u(r)=u$ and $\mu(\tau)=\mu$ are given (i.e., non-stochastic), then $A(r)$ satisties

$$
\begin{aligned}
\langle A(r)\rangle & =-\frac{1+Z}{u^{3}} \mu, \\
\left\langle A(\tau) A\left(\tau^{\prime}\right)\right\rangle & =\frac{1+Z}{u^{3}}\left(1-\mu^{2}\right) \delta\left(\tau-r^{\prime}\right),
\end{aligned}
$$

where the angle brackets denote averaging over the ensemble defined by all the realizations of $A$. Conoider following a particular electron using Eqs. (4). Suppose that the electron is observed to travel with velocity $u^{\prime}$ at $r=0$. Then $g\left(\mathbf{u}, r ; \mathbf{u}^{\prime}\right) d^{3} \mathbf{u}$ is the probability that the velocity of the electron at time $r$ is in the volume element $d^{3}$ te lesated at $\mathbf{u}$. In Appendix $A$, it is shown that this conditional probability $g$ satigfies Eq. (3). Thus the solution to Eq. (3) can be found by determining the distribution of a latge number of electrons obeying Eqe. (4) with initial conditions with $u(r=0)=\mathbf{u}^{\prime}$. Consequently, moments of $\mathrm{g}$ can be deternined by eusentite averages of the Langevin variables. For example, the current given by Eq. (3) may be found by

$$
\int d^{3} u u \mu g\left(u, \mu, \tau ; u^{\prime}, \mu^{\prime}\right)=\langle u(\tau) \mu(\tau)\} .
$$

Equations (4) may be integrated numerically by noting that

$$
\int_{r}^{r+\Delta r} A\left(r^{\prime}\right) d \tau^{r}
$$

should be picked from an ensemble with mean $-(1+Z) \mu \Delta r / u^{3}$ and variance $(1+Z)\left(1-\mu^{2}\right) \Delta r / u^{3}$ where $u$ and $\mu$ are the values of those variables at time $\tau$. As long as $\Delta r$ is sufficiently small, furtber details about the distribution of $A$ are unimportant.

Now Eqs. (4) are the equations solved in our earlier paper. ${ }^{2}$ This shows the exact equivaleace between the approsch adopted there and that employed in the present work. Because the Langevin equations describe the electron behavior in a slightly more physical manner, they often help in the interpretation of the solutions to the Boltzmann equation. This is especially true when some electrons run away. The Langevin equations are also very easy to solve numerically by a Mnnje-Carlo method (2s was done in Ref. 2), although their solution tends to be mucin more contiy than just solving Eq. (3) directly.

Equations (4) however may he easily solved analytically in the limit $u \rightarrow 0$ (this is equivalent to taking the limit $E \rightarrow 0$ ). Taking an ensemble average of the equationg, we obtain

$$
\begin{aligned}
\frac{d u}{d \tau} & =-\frac{1}{u^{2}}, \\
\frac{d(\mu)}{d \tau} & =-\frac{1+Z}{u^{3}}\{\mu\} .
\end{aligned}
$$


Note that is is not a stochastic variable in this limit. Consequently, the hierarchy of moment equations may be closed at this point. These are the slowing-down equations solved by Fisch and Boozer ${ }^{19}$ to give the current moment of electron distribution $\langle u \mu\rangle=\tau(\mu)$. This shows that the approach used in that paper is equivalent to solving the Boltzmann equation.

\section{ADJOINT METHOD}

The methods for solving the Boltzmann equation deacribed in the previous section all entai' a large amount of computation. (An exeeption is the limit $E \rightarrow$ 0 , when the ensemble-averaged Langevin equations can be solved analytically. ${ }^{19}$ ) The problem with these methods is that they are all capable of giving the electron distribution function $f_{1}$. Since, in many cases, we are only interested in specific moments of $f_{1}$, we may hope to reduce the computational requirements substantially by using a method that gives only those specific moments. Suppose we wish to determin' a particular moment of $f_{1}$, pamely

$$
H(\tau)=\int d^{3} \mathbf{u} h_{0}(u) f_{1}(u, \tau)
$$

(For instance, the current density would be given hy $h_{0}(u)=u_{\|}$.) Let us define the corresponding moment of the Green's function

$$
\cdot h\left(\mathbf{u}^{\prime}, \tau\right)=\int d^{\prime} \mathbf{u} h_{0}(\mathbf{u}) g\left(\mathbf{u}, \tau ; \mathbf{u}^{\prime}\right) .
$$

The moment: $H$ is theil given in terms of $h$ by

$$
H(\tau)=\int_{0}^{r} d \tau^{\prime} \int d q S\left(u, r^{\prime}\right) \cdot \frac{\partial}{\partial u} h\left(u, r-r^{\prime}\right) .
$$

What is needed is some method of calculating $h$ which doesn't involve baving to find $g$. This is provided by the adjoint formulation of Fisch. ${ }^{5}$ He shows that $h(\mathbf{u}, r)$ satisfies

$$
\frac{\partial}{\partial \tau} h+D^{*}(h)=0
$$

with $h(u, T=0)=h_{0}(u)$ and with the operator $D^{*}$ defined by

$$
D^{*} \equiv \frac{\partial}{\partial u_{\|}}+\frac{1}{t^{2}} \frac{\partial}{\partial u}-\frac{1+Z}{2 u^{3}} \frac{\partial}{\partial \mu}\left(1-\mu^{2}\right) \frac{\partial}{\partial \mu} \text {. }
$$

The singularity at the origin is handled by the boundary condition $h(\mathbf{u}=0, r)=$ 0 . The operatora $D$ and $D^{*}$ are adjoint operators, so that

$$
\int\left(h D(\Omega)-\int D^{*}(h)\right) d^{3} \mathbf{u}=0
$$


for all $f(u)$ and $h(u)$ satisifying $f(u \rightarrow \infty)=0$ and $h(u=0)=0$.

Similar techniques were introduced earlier by $\mathrm{Antonsen}$ and $\mathrm{Chu}^{20}$ and by Taguchi ${ }^{31}$ for the study of steady-state current drive. The significant improvements afforded by Ref. 5 are the ability to determine arbitrary moments of $f_{1}$ and the inclusion of the time-dependence of $f_{1}$. Both of these are important in the problem of current ramp-up.

From the relation between the two Green's functions $h$ and $g$, we see that $h$ has a simple unterpretation. Equations (3) and (4) describe the evolution of a group of electrons released at $r=0$ at velocity $u^{\prime}$. Let us suppose that we are interested in the current density so that $h_{0}(u)=u_{\|}$. Then $h\left(u^{\prime}, r\right)$ gives the mean current carried by those electrons a time $T$ later. How Eq. (8) works is easily seen by taking $u>1$ so that the electron only experiences the electric field. In the Baltzmann equation, the electrong have slowed down to $\mathbf{u}^{\prime}-r \mathbf{a}_{\|}$ at time $r$. Correspondingly in the adjoint equation, the initial condition $h_{0}$ is trangported in the reverse direction so that $h\left(u^{\prime}, r\right)=h_{0}\left(u^{\prime}-r \hat{u}_{\|}\right)$. Thus at time $r$, we are provided with information about the elrctrons in their curseat location.

Solving the Boltzmann equation by mengs of the adjoint formulation results in a great simplification of the problem. The adjoint equation (8) is an equation of equal complexity to the original Boltzmann equation (2). However, by solving Eq. (8) for a particular initial condition, we can find the corresponding moment of $f_{1}$ uting Eq. (7) for any driving term $S$.

The proof that $h$ is given by Eq. (8) is most easily carried out by assuming that Eq. (8) holds and then by proving that $h$ is related to the general Green's function $g$ by Eq. (6). Consid yr the equation for $g\left(\mathbf{u}, r^{\prime} ; \mathbf{u}^{\prime}\right)$,

$$
\left(\frac{\partial}{\partial \tau^{\prime}}+D\right) g\left(u, \tau^{\prime} ; u^{\prime}\right)=0 .
$$

We multiply this equation by $h\left(u, \tau-\tau^{\prime}\right)$, integrate over all velocity space, and use the adjoint relation between $D$ and $D^{*}$ to give

$$
\int d^{\prime} \mathbf{u} h\left(u, \tau-\tau^{\prime}\right) \frac{\partial}{\partial \tau^{\prime}} g\left(u, \tau^{\prime} ; u^{\prime}\right)+g\left(u, \tau^{\prime} ; u^{\prime}\right) D^{*}\left(h\left(u, \tau-\tau^{\prime}\right)\right)=0 .
$$

Substituting irom Eq. (8) and integrating in $\tau^{\prime}$ from 0 to $T$, gives

$$
\left.\int d^{\prime} \mathbf{u} h\left(u_{,} r-r^{\prime}\right) g\left(u, r^{\prime} ; u^{\prime}\right)\right|_{r^{\prime}=0} ^{+}=0 \text {. }
$$

If we evaluate this expression using the initial conditions for $g$ and $h$, we obtain Eq. (6).

Up until now, we have assumed that al the equations are solved in an infinite velocity domain. This is not a convenient formulation for numerical implementation where, necessarily, we wisb to solve equations in a finite dortain. 
Here we shall only solve Eq. (8) in a spherical domain $V$ such that $u<u_{b}$. We will choose $u_{6}$ to be sufficiently large that the interesting phygics where the electric field competes with the collisions happens inside $V$. Outside $V$ collisions may be ignosed and the electrons are merely freely accelerated by the electric field. We muat impose boundary conditions on 2 , the boundary of $V$. Again we follow the treatment given in Ref. 5 . We begin by noting that both Eq. (2) and Eq. (8) are hyperbolic in the $u$ direction. The boundary therefore divides into two pieces depending on whether the characteristics enter or leave the domain. We define $\Sigma_{\text {in }}$ (resp. $\Sigma_{\text {out }}$ ) as that portion of $\Sigma$ on which $-\mu-1 / v^{2}<0$ (resp. $>0$ ). The characteristics of Eq. (2) enter $V$ on $\Sigma_{i n}$ and leave on $\Sigma_{\text {out, }}$, while those of Eq. (8) enter $V$ on $\Sigma_{\text {out }}$ and leave on $\Sigma_{\text {ia }}$. Boundary conditions must be sperified where the characteristics enter the domain $V$. For $u_{b}$ sufficiently large, the solution beyand $\Sigma$ may be determined by ignoring collisions. Thus

$$
f_{1}(\mathbf{u}, \tau)=f_{1}\left(\mathbf{u}+r a_{\|}, 0\right)=0
$$

for $u$ on $\Sigma_{\text {in }}$ and

$$
h(\mathbf{u}, r)=h\left(\mathbf{u}-r \mathbf{a}_{\|}, 0\right)=h_{0}\left(\mathbf{u}-r \mathbf{u}_{\|}\right)
$$

for $u$ on $\boldsymbol{\Sigma}_{\text {out }}$. (If energy ocattering had been included in the collision opezator, the equations would revert to parabolic, and boundary conditions would bave to be specifind over the whole of $\Sigma$. However, there would be a boundary layer where the characteristics of the approximate equations are outgoing, and the boundary conditions here would only weakly affect the solution in the interior of $V$.)

Although Eq. (8) was derived under the simplifying assur, pirons that the electric feld was constant and the high-velocity form of collision operator is valid, the adjoint method as described in Ref. 5 applies equally well without such restrictive assumptions. Thus, the equation adjoint to Eq. (1) reads ${ }^{5}$

$$
\left(\frac{\partial}{\partial t^{\prime}}-\frac{q \mathrm{E}\left(t-t^{\prime}\right)}{m} \cdot \frac{\partial}{\partial \mathrm{v}}-C^{\mathrm{e}}\right) h\left(\mathrm{v}, t^{\prime} ; t\right)=q_{1}+q_{2} \varepsilon,
$$

where $C^{*}$ is the operator adjoint to $C$. Since the full linearized collision operator is self-adjoint, we have $C^{*}(h)=C\left(f_{m} h\right) / f_{m}$. Equation (g) is to be solved with the initial condition $h\left(v, t^{\prime}=0 ; t\right)=h_{0}(v)$. We restrict $f_{m} h$ to being orthogonal to 1 and $\varepsilon$ and $q_{1}$ and $q_{2}$ are chosen to ensure that this condition on $f_{m} h$ remains satisifed given that it is satisfied initjally. It can then be shown ${ }^{3}$ that

$$
\int d^{3} v f_{L}(v, t) h_{0}(v)=\int_{0}^{t} d t^{\prime} \int d^{3} \mathbf{v} S_{*}\left(v, t^{\prime}\right) \cdot \frac{\partial}{\partial v} h\left(v, t-t^{\prime} ; t\right),
$$

where $S_{n}(v, t)=S(v, t)+(q \mathrm{E}(t) / \mathrm{m}) \mathrm{S}_{\mathrm{m}}$. This equation will enable us to incorpc.ite the effects of a slowly varying electric field into our analysis. It also makes explicit the additive nature of those effectg due to the electric field alone 
(i.e., with $\left.S_{.}=(g \mathbf{E} / \mathrm{m}) f_{m}\right)$ and those effects due to the $\mathrm{rf}$ combined with the electric reld (i.e., with $\mathbf{S}$. $=\mathbf{S}$ ). Of course, the effects due to the electric field alone are well studied and give rise to phenstnena such as the Spitzer-Härm conductivity ${ }^{22}$ and runaways. ${ }^{16}$

\section{SOLUTIONS TO THE ADJOINT EQUATION}

Moments of the electron distribution $f_{1}$ can now be calculated by solving Eq. (8) with the corresponding initial and boundary conditions. In practice, this procedure still offers us too much iaformation. Both for a deeper understanding of the underlying physics and for easy implementation in numerical codes, the trick is to discover the few important functions by which the major effects can be described. In this section, we determine those functions needed for an accurate treatment of if current ramp-up.

Let us suppose that of flux is present only for some finite time. Electrons obeying Eq. (2) then eventually suffer one of twu fates. Either they run away under the influence of the electric field $u_{\|} \rightarrow-\infty$, or else they collapse into the electron bulk $u \rightarrow \mathbb{D}$. We classify these two groups of electrons as "runaway" (subscript r) and "stopped" (subscript a) respectively. In a real plasma, i.e., $T \neq 0$, even the bulk partieles will eventually fun away. However the time $t_{r}$ it takes for these bulk electrons to run away is exponentially large, i.e., $\log \mathfrak{r}_{r} \sim$ $\left(v_{r} / v_{\ell}\right)^{2}$. Our analysis is valid for times sbort compared with the bulk runaway time.

Runaways are very important in the calculation of the ramp-up efficiency because runaways gain energy at the expense of the poloidal magnetic field. Unlese they are lost, even a small number of runaways can greatly reduce the ramp-up efficiency. Runawajs may be defined as those particles with $u>u_{0}$ for $\tau \rightarrow \infty$ where $u_{0}$ is onme arbitrary positive speed. (The number of runaways is independent of $u_{0}$.) Therefore their number is given by Eq. (7) with $r \rightarrow \infty$ and $h_{0}(u)=1$ fo: $u>u_{0}$ and 0 otherwise. The Green's function for the runaway number is given by $R(\mathrm{u}) \equiv h(\mathrm{u}, \tau \rightarrow \infty)$ where $R$ obeys

$$
D^{*}(R(u))=0,
$$

with boundary condition $R(u)=1$ on $\Sigma_{\text {out- }}$ This function is the "runaway probability," the probability that an electron initially at $\mathbf{u}$ runs away under the combined infuence of the electric field and collisions.

Equation (11) was solved aumerically with the boundary at $u_{b}=10$. A term $\partial h / \partial r$ was in eluded on the left-hand side, and the resulting equation was integrated until $r=10 \mathrm{~J}$. A spherical $(u, \theta=\operatorname{atccos} \mu)$ grid was used with a mesh size of $500 \times 100$. The equation was integrated with an ADI (alternating direction implicit) echeme with a time step $\Delta r=0.01$. The same method was used to solve the osher equations given below. 
In Fig. 1, we plot $R(\mathrm{u})$ for $Z=1$. For $u<1, R$ is identically zero because the magaitude of the electrical force is less than that of the frictional force. One of the most important applications of these results is to drive current by lower-kybrid waves. In this case $\mathbf{S}$ is in the parallel direction and is localized near $u_{\perp}=0$. Therefore, we need only know $R\left(u_{\|}, u_{\perp}=0\right)$ which is plotted is Fig. 2 for $Z=1,2,5$, and 10. Frotn this plot, we see that we can effectively avoid the creation of sunaways by operating with waves whose phase velocities lie in the range $0<u_{\|} \lesssim 1.5$.

The next important quantity to determine is the current density carried by $f_{1}$. This is given (in units of $q n v_{r}$ ) by Eq. (7) with $h_{0}(u)=u_{\|}$. The Green's function for the current $j(u, r)$ is therr lore given by $\partial_{j} / \partial r+D^{*}(j)=0$ with initial condition $j(r=0)=u_{\|}$and boundar; condition $j=u_{\|}-r$ on $\Sigma_{\text {out }}$. This is the mean current (in units of $g v_{r}$ ) carried by an electron initially at velocity u. In Fig. 3(a), we plot $j(u, r)$ as a function of $\tau$ for $n=5 u_{i l}$ and $Z=1$. Because the presence of runatways leads to a secular behavior $(j \sim t)$ for large times, it is beipful to distinguish the current carried by stopper and runaway electrons. We write

$$
j(u, r)=(1-R(u)) j_{s}(u, r)+R(u) j_{r}(u, \tau)
$$

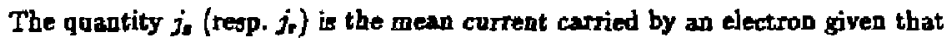
it eventually stops (resp. runs 2way). An electron at velocity $u$ mus away with probability $R(v)$. Thus it contributes $(1-R(u)) u_{\|}$to the stopped current and $R(u) u_{\|}$to the runaway current. These quantities are therefore the initial conditions to the adjoint equations for $(1-R) j_{\text {a }}$ and $R j_{r}$ respectively, so that

$$
\left(\frac{\partial}{\partial r}+D^{*}\right)(1-R)_{j}=\left(\frac{\partial}{\partial r}+D^{*}\right) R j_{r}=0,
$$

with $j_{s}(\tau=0)=j_{r}(\tau=0)=u_{\text {促 }}$ and $j_{\sigma}=j_{r}=\psi_{\|}-\tau$ on $\Sigma_{\text {oue }}$.

The stopped and runaway currents $j_{\text {, and } j}$ are plotted in $F$ igs. $3(b)$ and (c) for same case as Fig. 3(a). Evidently, $j$, vanishes for $\tau \rightarrow \infty$ (since the electrons cease to carry any current once they are stopped). The time it takes for the electrons to be stopped is of the order of $u$. Aesuming that this time is short compared to the time acale for the variation of the of lux $S_{\text {, we may replace }}$ $j_{0}(u r)$ by $W_{n}(u) \delta(r)$ where $W_{a}(u)=\int_{0}^{\infty} j_{s}(u, r) d \tau$. The equation for $W_{\theta}$ is obtained by integrating Eq. (8) over time to give

$$
D^{*}\left[(1-R(\mathbf{u})) W_{*}(\mathbf{v})\right]=(1-R(\mathbf{u}))_{\Psi_{\Pi}} .
$$

with $(t-R) W_{\mathrm{d}}=0$ on $\Sigma_{\text {act }}$. $W_{\text {a }}$ can be interpreted as the energy (in upits of $\left.m v_{r}^{2}\right)$ imparted to the electric field by an electron as it slows down. In Fig. $4(\mathrm{a})$, we plot $W_{\text {a }}(u)$ for $Z=1$. In the linit $u_{\|} \rightarrow \infty$, collisions are extremely weak, and all of th: kinetic erergy of the stopped particles goes into the electric field, i.e.,

$$
W_{s}\left(u_{\|} \rightarrow \infty, u_{\perp}=0\right) \rightarrow \frac{1}{2} u_{\|}^{2} .
$$


In the limit $u<1$, the electric field weakly perturbe the electron motion. Then, $W$, is given by the theory of steady-state current drive, ${ }^{19,20}$ and correctiong linear in the electric field are given by the hot conductivity. ${ }^{14}$ In our notation, these results may be aummarized by

$$
W_{s}(u<1, \mu)=\frac{\mu u^{4}}{5+Z}-\frac{\left(2+Z+3 \mu^{2}\right) u^{a}}{3(3+Z)(5+Z)} .
$$

This function is plotted in Fis. A(b). (An approximation to W, correct to order $\mathbf{u}^{10}$ is given in Appendix B.) This linenrized theory, however, is inapplicable for $u \sim 1$, and the behavior of this function is completely wrong for $u \geq 1$.

Let us now turn to the contribution of the nuaways to the current. The leading order contribution to $j_{r}$ in $-r$. Let us therefore write

$$
j_{r}(\mathbf{a}, \tau)=-r+j_{r o}(u)+j_{r}^{\prime}(\mathbf{u}, \tau) \text {, }
$$

where $j_{r}^{\prime}(\tau \rightarrow \infty) \sim 1 / \tau$ and $j_{r 0}$ may be interpreted as the eIfective starting velocity for the runaways; see Fig. $3(\mathrm{c})$. The function $j_{\mathrm{r}}$ is given by

$$
D^{*}\left(R(\mathbf{u}) j_{\circ}(\mathbf{u})\right)=R(\mathbf{u}) \text {, }
$$

with boundary condition $j_{r 0}=u_{\|}$on $\Sigma_{\text {orts }}$. This function is shown in Fig. 5. For $u>1$, the runaway electrons are only weakly perturbed by collisions so that $j_{r 0}(u) \approx u_{\|}$. Close to $u=1$, collinions hold back the runaway electrons and $j_{r o}(u)$ becomes large. However, it is not very important to know $j_{r o}$ and $j_{r}^{\prime}$ very accurately since they are usually dominated by the first term in Eq. (14). We will approxinate $j_{r o}(u)$ by $u_{\|}$and will ignore $j_{r}^{\prime}(u, r)$ to give $j_{r}(u, r)=u_{\|}-r$.

Finally, we can write the Green's function for the current in an expedient form as

$$
j(\mathrm{u}, \tau)=(1-R(\mathrm{u})) W_{\mathrm{a}}(\mathrm{u}) \delta(\tau)+R(\mathrm{u})\left(\mathrm{u}_{\|}-r\right) .
$$

In this form, it depends only on two sealar functions of $u$, namely $R$ and $W$. Approximate fits to these functions are given in Appendix B. An easy but important generalization is poasible here and that is to allow a loss mechanion for runaways. This is done by modifying suitably the term $u_{\|}-r$. For example, if the loss of nuawaya can be characterised by a loss time noen, this term should be multiplied by $\exp \left(-\pi / \eta_{0}\right)$.

Using this iormulation, many of her moments of $f_{1}$ may be found. For example, we may wish to know the mean perpendicular energy of the runaway particles $\varepsilon_{\perp r}$ (in units of $m v_{r}^{2}$ ) as they leave the integration region $V$. (The loss rate for runaways may depend on this quantity.) This is given by

$$
D^{\prime}\left(R(u) \varepsilon_{\perp_{r}}(\mathbf{u})\right)=0
$$

with $\varepsilon_{\perp r}=\frac{1}{3} u_{\perp}^{2}$ on $\Sigma_{\text {out }}$. (This rosult depends logarithmically on the value of $u_{6}$.) We have plotted this in Fig. 6. For thetrons with $u_{\|}>1$ and $u_{\perp}=0$, $\mathcal{E}_{1+}$ is about 3 . This reflects the necessity far the electrons to suffer appreciable pitela-angle scatteriog if they are to run away. 


\section{CIRCUIT EQUATIONS}

When rf epergy is injected into a tokamak, it induces a tux $\mathbf{S}$ of electrons in velocity space. The power deposited per unit volume is then given by

$$
p_{r}(t)=\int d^{4} v m S(v, l) \cdot v \text {. }
$$

Here, $p_{\boldsymbol{H}}, \mathrm{S}$, and the other intensive physical quantivies introduced in this section also depend on position r. For brevity, this dependence is not shown in the arguments to these quantities. This equation may be used in two ways. In detailed studies of if current ramp-up, based for instance on a ray-tracing model, we can estimate $\mathbf{S}(v, t)$ on each flux surface by solving a one- or two-dimensional Fokker-Planck equation. Equation (16) then gives us the power deposition, $p_{\mathrm{rf}}$. Alternatively, we ean take the experimental seasurements together with an energy balance of the if energy to give us an estimate of $p_{r f}$. This, together with an approtzimate knowledge of where in velocity space the $f$ f fux is localized, allows wo to decermine $\mathbf{S}$. In addition to causing power absorption, the flux $\mathbf{S}$ leads to numerous other effects, such as ef-driven currents, if-cohanced particle transport, etc. Here our primary concem is with the if-driven current. From Eq. (10), wo see that this enters additively to the ohmic cu:-rent so that the total current density is given by the constitutive relation

$$
J(t)=\sigma(t) E(t)+J_{M}(t),
$$

where $\sigma(t)$ is the Spitzer-Hairm conductivity ${ }^{22}$ for a Maxwellian plasma characterined by the beckground electron cemperature $T(t)$, and $J_{\mathrm{rf}}$ is the ff-driven current density. Here we bave asoumed that $\left|v_{r}\right|>v_{t}$ so that in the absence of any if we can ignore runaways. Incosporation of this effect merely requires the addition of the current carried by the Dreicer runaway electrons in Eq. (17).

The rf-driven current density is given by $\mathrm{Eq}$. (10) with the $h$ replaced by the current Green's function $j$ and with $\mathbf{S},=\mathbf{S}$. Let us begin by writing $j$ in unnormalized units. The form for $j$ given in $\mathrm{Eq}$. (15) will be sufficiently accurate for our purposes. Multiplying by $q v_{r}$ gives

$$
j(v, t)=\frac{q v_{r}}{\nu_{r}}(1-R(\mathrm{v})) W_{\mathrm{q}}(\mathrm{u}) \delta(t)+q R(\mathrm{u})\left(v_{\|}+\frac{q E}{m} t\right),
$$

where $u=v / v_{r}$. Here $j$ is uow a dimerional quantity, but $W_{s}$ and $R$ remain dimensianless functions of a dimensionless argument. In deriving this form for $j$ we assumed that $E$ and $n$ were constant. We now relax this constraint, allowing them both to vary on a time scale long compared to the runaway collision time $\nu_{r}^{-1}$. (Recall that $\mathbf{S}$ is aleo allowed to vary on the same time scale.) We can then write $j$ ag

$$
j\left(v, t-t^{\prime} ; t\right)=\frac{q v_{r}\left(t^{\prime}\right)}{v_{r}\left(t^{\prime}\right)}\left(1-R\left(u^{\prime}\right)\right) W_{s}\left(\mathbf{u}^{\prime}\right) \delta\left(t-t^{\prime}\right)
$$




$$
+q R\left(\mathrm{u}^{\prime}\right)\left(v_{\|}+\frac{q}{m} \int_{t^{\prime}}^{t} E(s) d s\right)
$$

where $u^{\prime}=v / v_{r}\left(t^{\prime}\right)$. The additional parametric argument $t$ here has the same meaning as in Eq. (9). In this form $j\left(v, t-t^{\prime} ; t\right)$ is the mean current carried by an electron at time $t$ given that it was traveling at velacity $v$ at time $t^{\prime}$. From Eq. (10), the if generated current density may now be written as

$$
J_{r s}(t)=\int_{0}^{t} d t^{\prime} \int d^{3} v \mathbf{S}\left(w, t^{\prime}\right) \cdot \frac{\partial}{\partial v} j\left(v, t-t^{\prime} ; t\right)
$$

In order to write $J_{r f}$ in a mare useful form, we frat define a runaway dengity $n_{r}$ (in electrons per unit volume). This is given by

$$
\frac{\partial n_{r}(t)}{\partial t}=\frac{1}{D_{r}(t)} \int d^{2} v S(v, t) \cdot \frac{\partial}{\partial u} R(u),
$$

with initial condition $n_{r}(t=0)=0$. Substituting Eq. (18) into Eq. (19), we obtain

$$
J_{\mathrm{rr}}(t)=J_{\mathbf{a}}(t)+J_{r}(t)
$$

where

$$
\begin{aligned}
J_{v}(t) & =\frac{q}{v_{r}(t)} \int d^{2} \mathbf{S}(\mathbf{v}, t) \cdot \frac{\partial}{\partial \mathbf{u}}(1-R(\mathbf{u})) W_{s}(\mathbf{u}), \\
\frac{\partial J_{r}(t)}{\partial t} & =\frac{q^{2}}{m} E(t) n_{r}(t)+q \int d^{2} \mathbf{v}(\mathbf{v}, t) \cdot \frac{\partial}{\partial \mathbf{u}} R(\mathbf{u}) u_{\|},
\end{aligned}
$$

with $J_{r}(t=0)=0$. In Eqs. (20) and (21), $u$ is normalized in terms of the runaway velocity at time $t, u=v / v_{r}(t)$. These equations allow the current to be calculated by characterizing the runaway population with just two state variable $n_{r}$ and $J_{r}$. Equations (16), (17), (20), and (21) suffice to give a detailed description of $\mathrm{f}$ current ramp-up. In thio form, Eq. (17) in suitable for gubstituting into a tranport or ray-tracing eode. Furtherriore, it would be easy to modify Eq. (20) to include a lose mechanism for the runawayg. Relativistic effects on the rupaways could be included in an approximate fasbion by limitiog $\left|J_{+}(t) / q n_{r}(t)\right|$ to $c$ the speed of light. Such effects could be treated in a more syatematic manner by modifying the term in large parentheses in Eq. (18) to read $v_{\|}(t)$ where

$$
\begin{aligned}
\vartheta_{\|}(t) & =p_{\|}(t) / m_{-}, \\
P_{\|}(t) & =m v_{\|}+\int_{t}^{t} q E(o) d t, \\
\gamma & =\sqrt{1+p_{\|}^{2}(t) / m^{2} c^{2}} .
\end{aligned}
$$

The resulting expression for $j\left(v, t-t^{\prime} ; t\right)$ is valid for $v_{\tau}^{2}<c^{2}$ and $v^{2} \ll c^{2}$. Unfortunately, this is a significantly more cumbersome expression from which 
to calculate $J_{\mathrm{rf}}$ because, in order to determine the stafe of the plasma at a particular ingtant, the entir runaway diatribution must he given (instead of just $n$, and $J_{r}$ ).

\section{APPLICATIONS}

The circuit equations written in Sec. $V$ aliow $u$ to explore how $J_{r}$ interacts with the electric field to yield ar efficient conversion of if energy into poloidal maznetic field energy. It is helpful to convert to extengive pbysical quantities by assuming that the plasma current is carried in a channel of area $A$ in which the plasma properties are approximately uniform. Thus, the total current is given by $I=A J$, the total if power deposited in the electrong by $P_{\text {in }}=2 \pi R_{0} A p_{\mathrm{rt}}$ (where $R_{0}$ is the tokamal major radius), the loop voltage by $V=2 \pi R_{0} E$, etc. The plasma current is again written as the sum of obmic and if contributions

$$
I=V / R_{\mathrm{gp}}+I_{\mathrm{rf}},
$$

where $R_{S_{p}}=2 \pi R_{0} / A \sigma$ is the plasma (Spitzer-Hârm) resistance. Faraday's law relates the rate of change of the current to the vollage

$$
V=-L \dot{I}+V_{\mathrm{ext}},
$$

where $L$ is the total plagma inductance, which for simplicity we shall take to be constant, $V_{\text {mxt }}$ is the voltage induced by the external coils (usually a combination of the ohmic windings and the vertical feld coils), and $I \equiv d J / d t$. Multiplying this equation by $I$ and substituting for $I$ from $\mathrm{Eq}$. (22) gives

$$
\dot{W}=P_{\text {ext }}+P_{\text {al }}-\frac{V^{2}}{R_{\mathrm{sp}}},
$$

where $W \equiv \frac{1}{2} L I^{2}$ is the poloidal field energy, $P_{\mathrm{nxt}} \equiv V_{\mathrm{ext}} I$ is the power car pled from the external circuits, and $P_{t 1} \equiv-V J_{r f}$ is the power coupled from twe if source into electromagnetic energy. This equation describe the energy balance for the poloidal magnetic field. The practical measure of the efficiency of current ramp-up is

$$
\frac{\dot{W}-P_{\mathrm{mat}}}{P_{\mathrm{rt}}}=\frac{P_{\mathrm{el}}-V^{2} / R_{\mathrm{gp}}}{P_{\mathrm{rt}}}
$$

where $P_{H}$ it the total rf power ivjected into the plasma. The if power absorbed by the electrons $P_{\text {in }}$ is related to $P_{r t}$ by $P_{i n}=\eta P_{r}$ where $\eta$ is the absorption factor. The determination of $\eta$ is beyond the scope of this paper, presumably it can be found by $\mathrm{r}$-t tracing theories or by a power belance. The overall picture of the flow of power in an experiment is as follows: $R f^{\text {power }} \boldsymbol{P}_{\mathrm{rf}}$ is injected into the machine. Of this a fraction $\eta$ is absorbed by the resonant electrons; the rest may be absorhed by the ions or by the vacuum vessel. A fraction $P_{\text {el }} / P_{\text {in }}$ of 
this power is then converted into electrom aboetic energy. $P_{\text {axt }}$ acts as another source of poloidal field energy, while the ohmic dissipation $V^{2} / R_{\mathrm{sp}}$ acts as a drain. From this discuasion, we see that $P_{\text {al }} / P_{\text {in }}$ describes the "ideal" efficiency of $f$ cursent ramp-up. The practical efficiency is expressible in terms of this efficiency, $\eta$, and $V^{2} / R_{S_{p}}$.

The determination of $P_{\mathrm{al}} / \boldsymbol{P}_{\mathrm{n}}$ from Eq. (21) is complicated by the presence of runawaye. Runuwaya are deleterious to the ramp-up efficiency sioce their current is in the same direction as $E$ and so they subtract from $\boldsymbol{P}_{\mathrm{a}}$. For efficient current ramp-up we must either avoid creating runaways by making sure $S$ is localized in that region of velocity space where the runaway probability $R$ is small (see Figs. 1 and 2), or else take steps to lose the runaways. We can approximately treat these casen by taking $P=0$ in $\mathrm{Eq}$. (21) to give

$$
\frac{P_{t-1}}{P_{l u}}=\frac{\int d^{3} \mathbf{u} S \cdot \partial W_{0} / \partial \mathbf{u}}{\int d^{2} \mathbf{u} S \cdot \mathbf{u}} .
$$

Since this involves the ratio of two integrals over $u$, the result is insensitive to tise detailed form of $\mathbf{S}$. In cases of practical interest, we may assume thai $\mathbf{S}$ is localized in u. Then, we have

$$
\frac{P_{\mathrm{al}}}{P_{\mathrm{in}}}=\frac{\hat{\mathbf{s}} \cdot \partial W_{\mathrm{s}} / \partial \mathbf{u}}{\hat{\mathbf{s}} \cdot \mathbf{u}},
$$

where $\mathbf{u}$ is the normalized velocity of the resonant electrons.

For lower-hybrid waves we have $\hat{\mathbf{S}}=\mathbf{u}_{\|}$and the waves interact with particles through the Landau resonance $\omega-k_{\|} v_{\|}=0$, where $\omega$ and $k_{\|}$are the wave frequency and parallel wave aumber. Furthermore, the typical perpendicular velocity of the resonant electrons equals the electron thermal velocity, so that $v_{\perp} \sim v_{t}<v_{\|}$. Thus Eq. (26) is to be evaluated with $u_{\|}=\omega / k_{\|} v_{r}$ and $u_{\perp}=0$. This gives

$$
\frac{P_{\mathrm{al}}}{P_{\mathrm{in}}}=\frac{\partial W_{\mathrm{a}} / \partial u}{u}
$$

This efficiency is plotted in Fig. $7(\mathrm{a})$. Approximate fits for this function are given in Appendix B.

On the other hand, for electron-cyclotron waves which interact through the Doppler-shifted cyclotron resonance $\omega-\dot{k}_{\|} v_{\|}=\Lambda$, where $\Omega$ is the cyclotron frequency and $t$ is the harmonic number, we bave $\hat{S}=\hat{u}_{\downarrow}$. In this case we evaluate Eq. (26) at $u_{\|}=(\omega-I \Omega) / k_{\|} v_{r}$ and $u_{\perp}=0$ to give

$$
\frac{P_{\mathrm{ll}}}{P_{\mathrm{iD}}}=\frac{\partial W_{s} / \partial u-\left(1 / u_{\|}\right) \partial W_{s} / \partial \mu}{u}
$$

which is plotted in 5ig. $7(\mathbf{b})$.

Usiog Equ. (27), it is possible to identify regions of high conversion efficiency of wave energy to dectric energy, given the rest riction of $R$ small. Additionally, if 
the ohmic losses, $V^{3} / R_{S p}$, are small, then by Eq. (25), we see that the conversion of wave energy to poloidal field energy can be of high efficiency. This, in fact, is what has been achieved on the PLT experirnent, where conversion efficiencies of over $25 \%$ have been reported."

An important practical consequence of the circuit equations derived here is that fagt ramp-up rates, i.e., large $\bar{I}$, are possible at high density. In fact, these fast ramp-up rates are neceasary for high energy conversion efficiencies at high density. This can be seen as follows: The efficiency, $P_{\mathrm{el}} / P_{\mathrm{in}+}$ is a function of the dimensionless parameter th, depending, in addition, oaly weakly on $Z$. For a given machine and a given wave phase velocity, the parameter $u_{\|}$depends only on the ratio, $E / n$; and the ramp-up rate, $\dot{I}$, dependu only on the dc electric field, $E$. Thus, the efficiency dependa only on the ratio of $i$ to $n$. It has been observed experimentally on the PLT experiment that bigh efficiency of converting rf energ: to magnetic field energy is possible at a low plasma density. Thus, we can predici '.'at a similar high efficiency is possible in the event that tbe density and the ramp-up rate are scaled up together. In fact, for large rampup rates, high density can actually be desirable in that it impedes the production of runaways. Note that this window of denired dengity for a given ramp-up rate is counter to our intuition derived from steady-state considerations, where the larger the density the less the current-drive efficiency.

There are several optimizations that one might wish to achieve in the rampup problern. Oue is to maximixe the energy conversion efficiency, $P_{\mathrm{ql}} / P_{\mathrm{ip}}$. A second is to minimize the ramp-up time, $T_{\text {ramp }} \equiv I / \hat{I}$. The minimization of capital costs for the if system, however, may demand that we minimize $P_{\mathrm{rf}}$, the if power required to ramp-up a given current.

We can express this more precisely with some convenient formulas. In the absence of the external source $V_{\text {ext }}$, the ramp-up rate may be written using Faraday's law as

$$
i \approx \frac{5 E}{\ln R_{0} / a} \frac{\mathrm{MA}}{\mathrm{g}},
$$

where a tolamak inductance $L \approx \mu_{0} R_{0}$ ln $R_{0} / a$ was assumed, and where $E$ is the de electric field in units of $\mathrm{V} / \mathrm{m}$. Note that the ramp-up rate depends linearly on $E$ and is almost independent of geometry $\left(\ln R_{0} / a \approx 1\right)$. The amount of dissipated if power required can than be written as

$$
P_{\mathrm{rf}} \approx \frac{1}{\eta} \frac{\frac{1}{2} L r^{2}}{T_{\mathrm{ramp}}} / \frac{P_{\mathrm{al}}}{P_{\mathrm{n}}}
$$

if we neglect both ohmic losses $\left(V^{2} / R_{\mathrm{sp}}\right)$ and the external source $P_{\text {ext. }}$. Thus, in extrapolating results to Larger tolameiks (higher $\frac{1}{2} L J^{2}$ ), we can majotain linear (in the required stured enerzy) power requirements, with the same ramp-up time and the same efficiencies, if the density scales linearly with $\dot{I}$ and hence with $I$. Here, the wave phase velocities also remain the same, and is the event 
of the same temperatures, the physics of the damping may be expected to be very similar, so that the percentage of incident of power that is absorbed, $\eta$, remains constant too.

For exsmple, using Fig. $7(\mathrm{a})$, we see that in PLT with $n \approx 2 \times 10^{12} \mathrm{~cm}^{-3}$, $T \approx 1 \mathrm{keV}, \omega / k_{\|} \approx 6 v ; \approx \frac{1}{4} c$, we find reported ramp-up tates of $\mathrm{I}=120 \mathrm{kA} / \mathrm{s}$, or $E \approx 24 \mathrm{~m} V / \mathrm{m}$. Here $u_{\|} \approx 1.4$ and ideal efficiencies of about $33 \%$ at $Z=1$ may be expected with little ronaway production, consiatent with experimental data. Also, consistent with the data would be somewhat higher $Z$, but then ouly if the confinement of runsways were not perfect.

For reactor-grade tokamaks, say $L \Gamma^{2} \approx 400 \mathrm{MJ}$ and $I \approx 10 \mathrm{MA}$, a rampup time which is louger than that in PLT is desirable in order to minimize $P_{r f}$ and the capital cost of the if system. For a $30 \mathrm{~s}$ ramp-up time, a density of $5 \times 10^{13} \mathrm{~cm}^{-3}$ renders the ratio $I / n$ as in the PLT experiment. Employing a similar spectrum of wave $\left(\alpha, / k_{\|}=\frac{1}{4} c\right)$ in a plaoms of tempetature also similar to the PLT experiment ( $T \approx 1 \mathrm{keV}$ ) implies a similar $\eta$ (about 0.7 ). Thus, using Eq. (29), we see that $P_{r r} \approx 40 \mathrm{MW}$ would be required.

To sumnarize the tradeoffs here, we note that while $P_{\mathrm{af}} / P_{\mathrm{ln}}$ is minimized by considering only the ratio $\bar{l} / n$, the minimization of $P_{\mathrm{rf}}$ requires the $T_{\mathrm{ramp}}$ be large. Thus, although very quick ramp-up rates are indeed achievable at bigh density, the capital costs for such a system are proportionately larger too. Balanciug the desires for a quick ramp-up against those for low capital custs (low $P_{\mathrm{rt}}$ ) points to a parameter range of moderate density. Efficient ramp-up is only achieved when, in addition to the above restrictions, the temperature ig moderate, gince at high temperatures, $V^{2} / R_{\mathrm{Sp}}$ losses, neglected in $\mathrm{Eq}$. (29), begin to dominate. The regime where these ohmic losse dominate may be identified by writing

$$
\frac{V^{2}}{\lambda_{s_{p}}} \approx \frac{L \Gamma^{2}}{T_{\text {rmp }}} \frac{L / R_{S_{p}}}{T_{\text {ramp }}}
$$

These losses represent only small corrections when $V^{2} / R_{\mathrm{Sp}} \ll P_{\mathrm{rt}}$, or, using Eq. (29), when

$$
\frac{T_{\text {ramp }}}{L / R_{S_{\mathrm{p}}}}>\frac{P_{\text {el }}}{P_{\mathrm{ri}}} \text {. }
$$

For the reactor-grade example, $P_{\mathrm{al}} / \boldsymbol{R}_{\mathrm{t}} \approx \frac{1}{3}, \eta \approx 0.7$, the above inequality requires that the ramp-up time be longer than about $\frac{1}{4}$ of the $L / R_{9 p}$ time. This restricts the temperature to somewhat less than $2 \mathrm{keV}$.

Restricting the temperature during a period of intense of injection (perhaps $40 \mathrm{MW}$ ) requires a small heat confinement time during the start-up operation. In the above example, this may be as small as $\mathbf{3 0} \mathrm{ms}$. Poor confinement during the start-up phaso may be helpful from the standpoint of runaway buildup too. Even a small percentage $(\sim 1 \%)$ of reverse runaway ${ }^{24}$ can seriougly izapede ramp-up if the runzways are well confined. If the runaways are poorly confined. then higher percentage may be tolerated, allowing higher ramp-up rates and, consequeutly, higher energy conversion efficiencies for a given density. 
We are led thus to the following typical picture of if ramp-up for pulsed tolsamak operation. Start-up can proceed in a low density plasma ${ }^{\text {33 }}$ where the if power is also used to initiate the planma. Density and if power, and the rampup rate, are increased concomitantly as the plasma is brought to intereating densities $10^{13}-10^{-4} \mathrm{~cm}^{-3}$. During this phase, the temperature is purposefully kept low, possibly through a deliberate degradation of the confinement of both runaway and thermal electrong. Hence the current is progranmed to reach a large value prior to the density, and both reach large values prior to the temperature. The final step, in which the reactor is brought to reactor-grade temperature, occurs after the currest is ramped up and as a result of ceasing the deliberate degradation of confinement.

\section{vII. CUNCLUSIŨNS}

In this paper we have written down a set of circuit equations that describe the dynamics of an rifdriven piaswion. ' $\because$ aniving at these circuit equations, we gysteraatically introduced approximetions with a goal of characterizing the driven plasma by a small number of functions of few variabies that retain the essential physics. Greater aicuracy, posoible at the price of more complex circuit equation, may be obtairied as a natural extension of the development here. The identification and calculation here of a minimal set of transport functions, however, provided a suitable and manageable description for a large class of important problems.

The calculations of the runaway function $R$ and of the energy conversion function $W$, together pinpoint the preferred region for tokamal ramp-up operation. These functions depead only on the dimensionless parameter $u$. The separate contributions of runaway and stopped currents may be described using these functions of a single variable. The constitutive relations thus obtained are given by Eq3. (21). These equations are in a form both suitable for implementation in a transport code and amenable to obvious modiffcation in the event that more complex runaway models are desired.

There are reveral caveats to bear in mind in using these formulas. First, the time scale for variation of the dc electric fields has been assumed long compared to other scales of interent, such as the particle deceleration times. A violation of this scale separation would affect the normalizations through y. . Second, knowledge of the if spectrum is unlikely to be complete. This knowledge is neceosary to give $\mathbf{S}$, the rf-induced liux. Even if the incident if energy is followed by ray-tracing codes, it remaina poraible that other waves may be present. Thase other waves might ariee either due to aymmetries in the particle distribution functions of due to nonlinear effects astociated with the incident spectrum. Thiri, particle transport acrom feld lines was neglected in comparison to the efiects along field lines. The neglect of these effects is passible for stopped electrons if they are stopped before they reach a fux aurface with sigaificantly 
different corditiuns ( $v_{r}$ different). For runaway electrons, these effects are always important is that they provide a model for the runaway loss. As discussed after Eqs. (21), $\mathrm{s}^{*} \mathrm{~h}$ model may be included through a natural modification of Eq. (E:.). In the absence of one particularly compelling model, at present, for runaway loss we bave left the modification of Eq. (21c) as an open issue.

Finally, we slould note that some of the moat powerful conclusions of this paper occor in certain opecial cases. It is often the case that the if spectrum is not only known, but also localized, which enables a particularly simple evaluation of the conversion efficiency, as in Eqs. (27). In the event of moderate electric Selds, or spectra localized at moderate phase velocities, it may be that $R=0$ (no runaway production), and an accurate runaway loss model would not be ocded. In the event that runaways are confined well, the spectrum must be chosen carefully io assure that $R=0$.

\section{ACKNOWLEDGMENTS}

This work was supported by the United Stater Department of Energy under Contract DE-AC02-76-CHO-3073. 


\section{APPENDIX A. LANGEVIN EQUATIONS}

Here we show that the conditional probability distribution $g\left(\mathbf{u}, \tau ; \mathbf{u}^{\prime}\right)$ for Eqs. (4) satiofies Eq. (3). The derivation follows those given in Refs. 18 and 25. Because the process described by Eqs. (4) is a Markoff process, $g$ satisâes the Smolucowski equation ${ }^{15}$

$$
g\left(\mathbf{u}, \tau+\Delta r ; u^{\prime}\right)=\int d^{3} \mathbf{u}^{\prime \prime} g\left(\mathbf{u}, \Delta r ; \mathbf{u}^{\prime \prime}\right) g\left(\mathbf{u}^{\prime \prime}, r ; \mathbf{u}^{\prime}\right)
$$

for ail $\tau>0$ and $\Delta \tau>0$. Let us define

$$
r(\mathbf{w}, \mathbf{u}, \Delta \tau) \equiv g\left(\mathbf{u}+w_{1} \Delta r_{;} \mathbf{u}\right) .
$$

Subtracting $g\left(\mathbf{u}, \tau ; \mathbf{u}^{\prime}\right.$; from $\mathrm{Eg}$. (Al) gives

$$
\begin{aligned}
g\left(\mathbf{u}, \tau+\Delta \tau ; \mathbf{u}^{\prime}\right)-g\left(\mathbf{u}, \tau ; \mathbf{u}^{\prime}\right)=\int d^{\prime \prime} \mathbf{u}^{\prime \prime} & {\left[\boldsymbol{r}\left(\mathbf{u}-\mathbf{u}^{\prime \prime}, \mathbf{u}^{\prime \prime}, \Delta \tau\right) g\left(\mathbf{u}^{\prime \prime}, \tau ; \mathbf{u}^{\prime}\right)\right.} \\
& \left.-r\left(\mathbf{u}-\mathbf{u}^{\prime \prime}, \mathbf{u}, \Delta \tau\right) g\left(\mathbf{u}, \tau ; \mathbf{u}^{\prime}\right)\right],
\end{aligned}
$$

where, because of the normalization condition for probabilities, the second term in the integral may be reduced to $g\left(u_{1} r ; u^{\prime}\right)$. If we change the variable of integration to $w=u-u^{\prime \prime}$, the right-hand side of Eq. (A2) becomes

$$
\int d^{3} \mathbf{w}\left[r(w, u-w, \Delta r) g\left(u-w, r ; u^{\prime}\right)-r(w, u, \Delta r) g\left(u, r ; \mathbf{u}^{\prime}\right)\right] .
$$

For small $\Delta r$, the function $r(w, u, \Delta r)$ is highly localized about $w=0$. We may therefore expand the first term in the integral, assuming that $w$ is much smaller than $u$, to give

$$
\begin{aligned}
r(w, u-w, \Delta r) g\left(\mathbf{u}-w, r ; \mathbf{u}^{\prime}\right) \approx & r(w, u, \Delta \tau) g\left(\mathbf{u}, \tau ; \mathbf{u}^{\prime}\right) \\
& -\mathbf{w} \cdot \frac{\partial}{\partial \mathbf{u}} r(\mathbf{w}, \mathbf{u}, \Delta \tau) g\left(\mathbf{u}, \tau ; \mathbf{u}^{\prime}\right) \\
& +\frac{1}{2} \mathbf{w w}: \frac{\partial^{2}}{\partial \mathbf{u} \partial \mathbf{u}} r(\mathbf{w}, \mathbf{u}, \Delta \tau) g\left(\mathbf{u}, r ; \mathbf{u}^{\prime}\right) .
\end{aligned}
$$

Using this approximation in Eq. (A2), integrating by parts, dividing by $\Delta r$ and taking the limit $\Delta r \rightarrow D$, we find as the equation for $g\left(u, r ; u^{\prime}\right)$

$$
\frac{\partial}{\partial \tau} g=\frac{\partial}{\partial \mathbf{u}} \cdot \mathbf{A g}+\frac{\partial^{2}}{\partial \mathbf{u} \partial \mathbf{u}}: \mathbf{B g},
$$

where

$$
\begin{aligned}
& \mathbf{A}(\mathbf{u})=\lim _{\Delta r-0}-\frac{\langle\Delta u\rangle}{\Delta \tau}, \\
& \mathbf{B}(\mathbf{u})=\lim _{\Delta r \rightarrow 0} \frac{\langle\Delta u \Delta u\rangle}{2 \Delta \tau},
\end{aligned}
$$


and

$$
\begin{aligned}
(\Delta \mathbf{u}) & =\int w r(w, u, \Delta r) d^{s} w, \\
\langle\Delta u \Delta u) & =\int w w r(w, u, \Delta r) d^{3} w .
\end{aligned}
$$

Thus $\{\Delta u)$ is the average value of $u(r+\Delta r)-u(r)$ given that $u(r)=u$ (and similarly for $(\Delta u \Delta u))$. These quantities may be calculated directly from Eqs. (4) assuming that $\Delta t$ is sufficiently small that $u$ does not change appreciably. We then obtain

$$
\begin{aligned}
& \langle\Delta u\rangle=-\int_{\tau}^{\tau+\Delta \tau}\left\langle\frac{1}{u\left(\tau^{\prime}\right)^{2}}+\mu\left(\tau^{\prime}\right)\right\rangle r^{\prime} \\
& \approx-\left(\frac{1}{u^{2}}+\mu\right) \Delta r \\
& \langle\Delta \mu\rangle=\int_{r}^{r+\Delta r}\left(A\left(\tau^{\prime}\right)-\frac{1-\mu\left(r^{\prime}\right)^{2}}{u\left(\tau^{\prime}\right)}\right) d \tau^{\prime} \\
& \approx-\left(\frac{1+Z}{u^{3}} \mu+\frac{1-\mu^{2}}{u}\right) \Delta r \\
& (\Delta \mu \Delta \mu)=\int_{r}^{r+\Delta r} \int_{r}^{r+\Delta r}\left(A\left(r^{\prime}\right) A\left(r^{\prime \prime}\right)\right) d r^{\prime} d r^{\prime \prime}+O\left(\Delta r^{2}\right) \\
& \approx \frac{1+Z}{u^{3}}\left(1-\mu^{2}\right) \Delta r \\
& (\Delta u \Delta u)=(\Delta u \Delta \mu)=(\Delta \mu \Delta t)=O\left(\Delta \tau^{2}\right) .
\end{aligned}
$$

Here we have made use of the properties of $A$ given in Eqs. (5). Writiog Eq. (A3) in spherical coordinates and substituting for the non-zero components of $A$ and 8, we obtain

$$
\begin{aligned}
\frac{\partial}{\partial \tau} g & =\frac{1}{u^{2}} \frac{\partial}{\partial u} u^{2} A_{u g} g+\frac{\partial}{\partial \mu} A_{\mu} g+\frac{\partial^{2}}{\partial \mu^{2}} B_{\mu \mu} g \\
& =\frac{\partial}{\partial u \|} g+\frac{1}{u^{2}} \frac{\partial}{\partial u} g+\frac{1+Z}{2 u^{3}} \frac{\partial}{\partial \mu}\left(1-\mu^{2}\right) \frac{\partial}{\partial \mu} g .
\end{aligned}
$$

This is the same equation as Eq. (3). Furthermore, from the deffnition of $g$ as a conditional probability, the initial condition for Eq. (A4) is also the same as for Eq. (3), namely; $q\left(u, r ; \mathbf{u}^{\prime}\right)=\delta\left(u-\mathbf{u}^{\prime}\right)$.

\section{APPENDIX B. NUMERICAL FITS}

In this appendix, we give approximations for some of the important functions we have calculated. These are suitable for incorporating into modeling codes. The approximations were found by choosing a suitable analytic form containing 
saveral undetermined coefficients and adjugting those coeficients in order to mininize the maximum relative error. The technique for carrying out this prosedure is described in Hastings' classic work. ${ }^{20}$ The Gits were made to the nunerical data presented in Sec. IV. This data itself contains errors due to the numerical methods used. The main source of error is due to the finite size of the numerical mesh and it is estimated that this intioduces errors on the order of a percent. However, near $\boldsymbol{x}=0$, the relative error in the numerical data for $W$, and its derivative becomes large because $W_{s}=O\left(u^{4}\right)$. Thus for $u<0.5$, the fits were made using the following analytical approximation instead of the pumerical data:

$$
\begin{aligned}
W_{s}= & \frac{\mu u^{4}}{Z+5}-\frac{\left(2+Z+3 \mu^{2}\right) u^{8}}{3(3+Z)(5+Z)} \\
& +\frac{2\left(\left(24+19 Z+3 Z^{2}\right) \mu+(9+Z) \mu^{3}\right) u^{8}}{(3+Z)(5+Z)(7+3 Z)(9+Z)} \\
& -\frac{\left(\begin{array}{c}
10+1+1804 Z+1160 Z^{2}+316 Z^{3}+30 Z^{4} \\
\left.+10\left(147+497 Z+101 Z^{2}+21 Z^{3}\right) \mu^{2}+5(9+Z)(13+3 Z) \mu^{4}\right) u^{10}
\end{array} .\right.}{5(2+Z)(3+Z)(5+Z)(7+3 Z)(9+Z)(13+3 Z)} .
\end{aligned}
$$

This result was obtained by solving Eq. (12) for small $u$ uging MAcsYMA. ${ }^{27}$ (The first two terms in this expansion are those derived by Fisch. ${ }^{14}$ )

For each of the functions approximated, we give the analytic form of the approximation, the range in which it is valid, a table of coefficients, and the maximum relative ersor. The approximation should not be used outside the range given. Alsa, bote that the relative error quoted is the error in littiog the approximation to the numerical data which itself is in error by about a percent.

For $\mu=1$ and $1.4<u<8$, the runaway probability $R$ is approximated by

$$
R(u, \mu=1)=\exp \left(\frac{\sum_{i=0}^{3} a_{i}(u-1)^{i}}{\sum_{i=1}^{3} b_{i}(u-1)^{i}}\right),
$$

where $b_{1}=1$ and the otber coefficients $a_{i}$ and $b_{i}$ are given in Table I. The maximum relative error is $1 \%$. For $\mu=1$ and $1<u<1.4$, the same approximation may be used with small absolute error but large relative error. For $u<1$ and all $\mu$ we bave $R=0$ identically.

For $\mu=1$ and $0<u<5$, the energy imparted to the electric field $W$, by stopped electrons is approximated by

$$
W_{,}(u, \mu=1)=\frac{\sum_{i=0}^{4} a_{i} u^{2 i}}{\sum_{i=0}^{j} b_{i} u^{2 i}},
$$

where $b_{0}=1$ and the other coefficients $a_{\text {, }}$ and $b_{i}$ are given in Table II. The maximium relative error is $2 \%$. For $\mu=-1$ and $0<u<1, W$; is approximated by

$$
W_{\sigma}(u, \mu=-1)=\sum_{i=2}^{5} a_{i} u^{2 i},
$$


where the coefficients $a_{i}$ are given in Table III and the maximium relative error is $1.5 \%$.

For $\mu=1$ and $0<u<5$, the function $\left(\partial W_{s} / \partial u\right) / u$ is is approximated by

$$
\frac{1}{u} \frac{\partial}{\partial u} W_{s}(u, \mu=1)=\frac{\sum_{i=1}^{T} a_{i} u^{2 i}}{\sum_{i=0}^{3} \frac{b_{i} u^{2 i}}{2}}
$$

where $b_{0}=1$ and the other coefficients $a_{i}$ and $b_{i}$ are given in Table IV. The maximium relative error is $5 \%$. For $\mu=-1$ and $0<u<1,\left(\partial W_{s} / \partial u\right) / u$ is approximated by

$$
\frac{1}{\tau} \frac{\partial}{\partial u} W_{s}(u, \mu=-1)=\sum_{i=1}^{t} a_{i} u^{2 i},
$$

where the coeficieats $a_{i}$ are given in Table $V$ and the maximium relative error is $3 \%$. 


\section{REFERENCES}

IN. J. Fisch, Phys. Fev. Lett. 11, 873 (1978).

${ }^{2}$ N. J. Firch and C. F. F. Karney, Phys. Rev. Lett. 54, 897 (1985).

${ }^{3}$ C. F. F. Karney, N. J. Fisch, and F. C. Jobes, Princeton Plasma Physics Laboratory Report, PPPL-2152 (1984).

1F. C. Jobes, S. Bermabei, T. K. Chu, W. M. Hooke, E. B. Meservey, R. W. Motloy, J. E. Stevens, and S. E. von Goeler, Princeton Plasma Physics Laboratory Report, PPPL-2200 (1985).

${ }^{5}$ N. J. Fisch, Princeton Plasma Physics Laboratory Report, PPPL Ż03 (1985)

${ }^{\circ}$ R. W. Harvey, J. C. Riordan, J. L. Luxon, and K. D. Marx, Proc. 4th Topical Conf, on Rudio Frequency Plasma heating, Austin, Texas (1981). Paper C9.

${ }^{\top}$ C. S. Liu, Z. G. An, D. A. Boyd, Y. C. Lee, L. Muschietti, K. Appert, and J. Vaclavit, Comments Plasma Phys. Contr. Fusion T, 21 (1982).

${ }^{8}$ L. Muschietti, J. Vaclavik, and K. Appert, Plasma Phys. 24, 987 (1982).

${ }^{9}$ Z. G. An, C. S. Liu, Y. C. Lee, D. A. Boyd, L. Muschietti, K. Appert, and J. Vaclavik, Phys. Fluids 25, 997 (1982); 26, 345 (1983).

${ }^{10} \mathrm{~K}$. Appert, A. H. Kritz, S. Succi, and J. Vaclavik, Proc. 11th European Conf. on Contr. Fusion and Plasma Phys., Aachen (1983), Part 1, 329.

${ }^{11} \mathrm{~K}$. Borrass and A. Nocentini, Plasma Phys. and Contr. Fusion 28, 1299 (1984).

${ }^{12}$ C. S. Liv, V. S. Cban, and Y. C. Let, General Atomic Technologies Report GA-A17915 (1985).

${ }^{13}$ D. F. H. Start, Plasma Phys, 25, 793 (1983).

14N. J. Fisch, Phys. Fluids 28, 245 (1985).

${ }^{18}$ C. F. F. Karney and N. J. Fioch, Phys. Fluids 28, 116 (1985).

${ }^{16} \mathrm{H}$. Dreicer, Phys. Rev. 117, 329 (1960).

${ }^{17}$ C. F. F. Karney and N. J. Fioch, Phys. Fluids 22, 1817 (1979)

${ }^{19}$ M. C. Wang and G. E. Uhlenbeck, Rev. Mod. Phys. 17, 323 (1945) in Selected Papero on Noise and Stochastic Processes, edited by N. Wax (Dover, New York, 1954).

${ }^{19}$ N. J. Fisch and A. H. Boozer, Phys. Rev. Lett. 45, 720 (1980).

${ }^{20}$ T. M. Antonsen and K. R. Chu, Phys. Fluids 25, 1295 (1982).

${ }^{21}$ M. Taguehi, J. Pbya. Soc. Jpn. 62, 2035 (1983).

${ }^{27}$ L. Spitzer and R. Hïrm, Phyo. Rev. 89, 977 (1953)

${ }^{23}$ F. C. Jobes, J. Sterens, R. BeIl, S. Bernabei, A. Cavallo, T. K. Chu, S. Cohen,

B. Denze, P. Efthimion, E. Hinnov, W. Hooke, J. Hosea, E. Mazzucato, R. McWilliams, R. Motley, S. Suckewer, G. Taylor, J. Timberlake, S. von Goeler, and R. Wilson, Phya. Rev. Lett. 52, 1005 (1984).

${ }^{24} \mathrm{D}$. C. Eder and E. J. Valeo, private communication (1984). 
${ }^{25}$ E. M. Lifshitz and L. P. Pitaevslii, Physical Kinetics (Pergamon- Press, Oxford, 1981).

${ }^{26}$ C. Hastings, Approsimations for Digital Computers, Princeton University Press (Princeton, 1955).

${ }^{27}$ The Matblab Group, MACSYMA Reference Manuad, Version 10, Laboratory for Computer Science, Massachusetts Institute of Technology (1983). 
TABLE I. Coeficieats for approximation to $R(u, \mu=1)$.

\begin{tabular}{|c|c|c|c|c|c|c|}
\hline $\bar{Z}$ & $a_{0}$ & $\boldsymbol{a}_{1}$ & $\overline{a_{2}}$ & $a_{3}$ & $\overline{b_{2}}$ & $\overline{b_{3}}$ \\
\hline $\boldsymbol{1}$ & -3.68043 & 4.23913 & -4.55894 & -0.39755 & -1.22774 & 1.4145 \\
\hline 2 & -4.97636 & -16.69015 & 0.83188 & 0.21737 & 6.84615 & -0.98649 \\
\hline 5 & -4.27687 & -4.33629 & 0.30338 & 0.05697 & 3.21315 & -0.47749 \\
\hline 10 & -4.94597 & -1.53482 & 0.10112 & 0.03087 & 2.45288 & -0.368 \\
\hline
\end{tabular}

TABLE II. Coefficients for approximation to $W_{s}(u, \mu=1)$.

\begin{tabular}{ccccccc}
\hline$Z$ & $a_{2}$ & $a_{3}$ & $a_{4}$ & $b_{1}$ & $b_{2}$ & $b_{3}$ \\
\hline 1 & 0.16612 & -0.01495 & 0.00775 & 0.37136 & 0.02240 & 0.01645 \\
2 & 0.14200 & -0.04048 & 0.01145 & 0.12253 & 0.00384 & 0.02440 \\
5 & 0.09880 & -0.05152 & 0.01113 & -0.19484 & 0.00559 & 0.02362 \\
10 & 0.06537 & -0.03895 & 0.00738 & -0.32456 & 0.02797 & 0.01526 \\
\hline
\end{tabular}

TABLE III. Coeficients for approximation to $W_{s}(u, \mu=-1)$.

\begin{tabular}{ccccc}
\hline \hline$Z$ & $a_{2}$ & $a_{3}$ & \multicolumn{1}{c}{$a_{4}$} & $a_{5}$ \\
\hline 1 & -0.16483 & -0.13420 & 0.15346 & -0.24314 \\
2 & -0.14186 & -0.09297 & 0.06661 & -0.12870 \\
5 & -0.09975 & -0.04781 & 0.00606 & -0.03545 \\
10 & -0.06651 & -0.02797 & -0.00247 & -0.00934 \\
\hline \hline
\end{tabular}

TABLE IV. Coelicients for approximation to $\left(\partial W_{s} / \partial u\right) / u$ for $\beta=1$.

\begin{tabular}{ccccccc}
\hline$Z$ & $a_{1}$ & $a_{2}$ & $a_{3}$ & $\overline{b_{1}}$ & $\overline{b_{2}}$ & $\overline{b_{3}}$ \\
\hline 1 & 0.66445 & -0.36032 & 0.07328 & 0.17769 & -0.25452 & 0.07278 \\
2 & 0.56760 & -0.38984 & 0.08634 & -0.04019 & -0.24673 & 0.08508 \\
5 & 0.39909 & -0.32879 & 0.07670 & -0.28281 & -0.16275 & 0.07436 \\
10 & 0.27028 & -0.23261 & 0.05272 & -0.39140 & -0.07526 & 0.04881 \\
\hline
\end{tabular}

TABLE $\checkmark$. Coefficienta for approximation to $\left(\partial W_{s} / \partial u\right) / u$ for $\mu=-1$.

\begin{tabular}{ccccc}
\hline$Z$ & $a_{1}$ & $a_{2}$ & $a_{3}$ & $a_{4}$ \\
\hline 1 & -0.63673 & -1.39960 & 3.37662 & -4.23684 \\
2 & -0.55777 & -0.80763 & 1.43144 & -2.03866 \\
5 & -0.39704 & -0.33911 & 0.23607 & -0.51011 \\
10 & -0.26600 & -0.17342 & 0.01896 & -0.13349 \\
\hline \hline
\end{tabular}




\section{FIGURES}

FIG. 1. The runaway probability $R(u)$ for $Z=1$. Parts (a) and (b) shrew $R$ on two different scales. In (a) the contours are equally opaced at intervals of 0.05 . In (b) the lowest 7 contours are geometrically spaced at intervals of $10^{1 / 3}$ between $10^{-3}$ and $10^{-1}$; the remaining contours are equally spaced at intervals of 0.05 as in (a).

FIG. 2. $R\left(u_{\|}, u_{\perp}=0\right)$ for $Z=1,2,5$, and 10 .

FIG. 3. The current $j(\mathbf{u}, r)$ for $\mathbf{a}=5 \hat{u}_{\mid j}$ and $Z=1$; ( $\mathrm{B}$ ) the total curreat $j$; (b) the stopped current $j_{s}$; (c) the runaway current $j_{r}$. For $u=5 \hat{u}_{\|}$, approximately $32 \%$ of the electrons run away.

FIG. 4. The energy imparted to the electric field by the stopped perticles $W_{s}(\mathrm{~d})$ for $Z=1$. The innermost contours are equally spaced at intervals of 0.005 between -0.05 and 0.05 . The remaising contours are equally spaced at interials of 0.05. Part (a) showe the results of numerically solving Eq. (12); part (b) shows $W$, from the hot-conductivity theory Eq. (13).

FIG. 5. The function $j_{r o}(u)$ for $Z=1$. The contours are equally spaced at intervals of 0.25 .

FIG. 6. The perpendicular euergy of the runaways $\varepsilon_{\perp-}(u)$ for $Z=:$. The contours are equally spaced at intervals of 0.5 .

FIG. 7. Efficiency for lower-hybrid current drive (a) and for electron-cyclotron current drive (b) from Eq̣. (27). 
* 85T0062
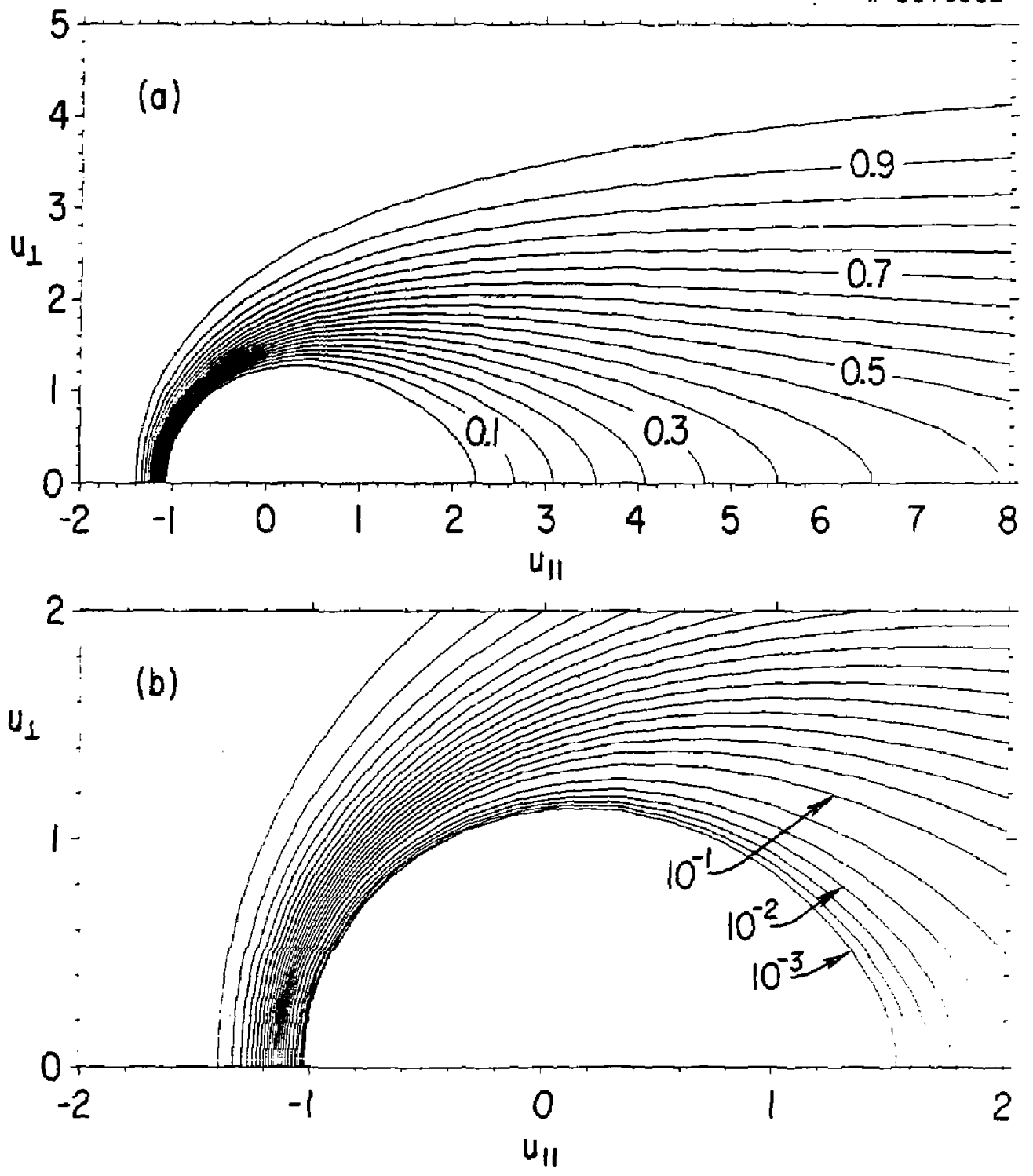

Fig. 1 


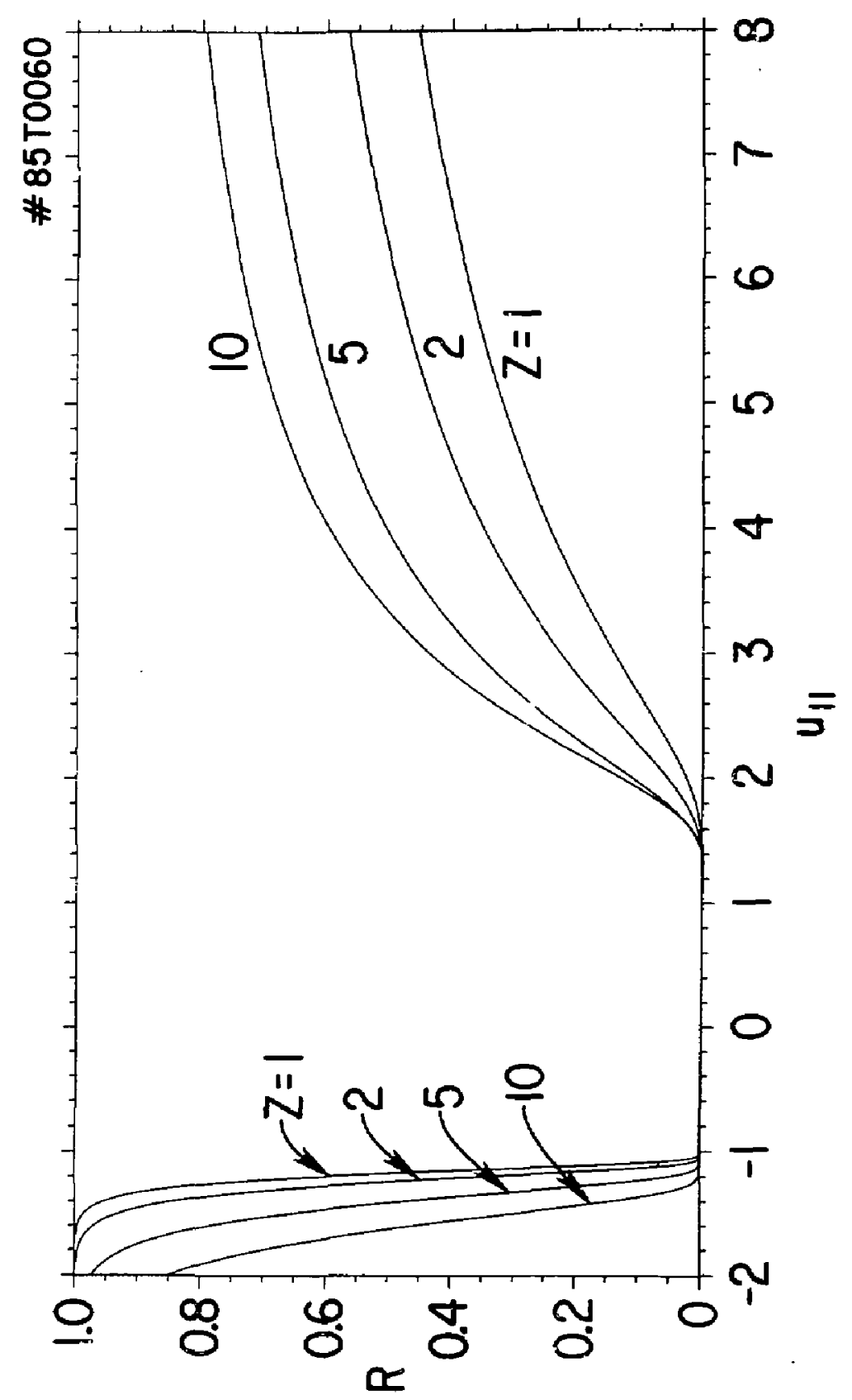

$\stackrel{N}{\infty}$ 

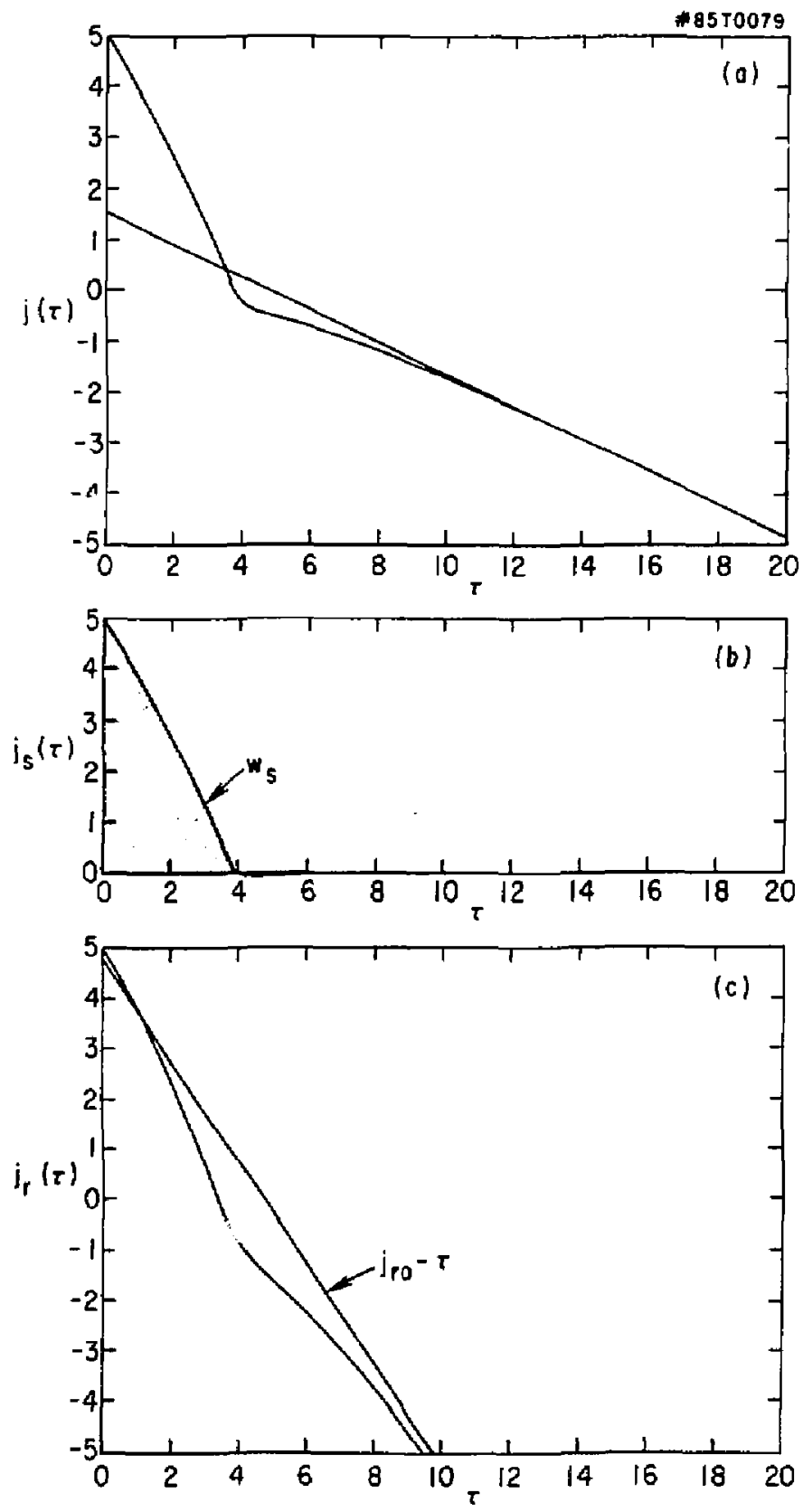

FIg. 3 
* 85T0058
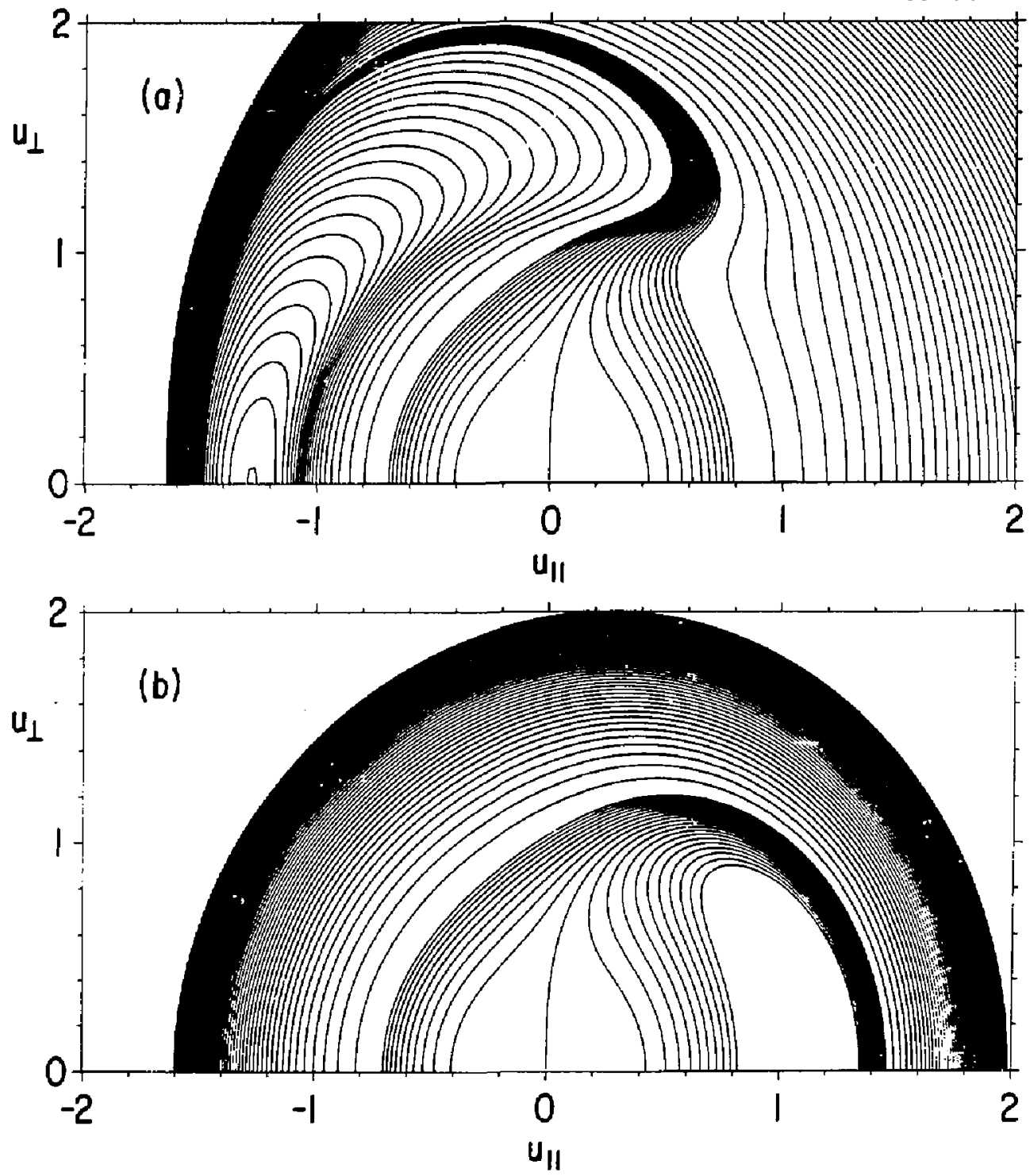

Fig. 4 


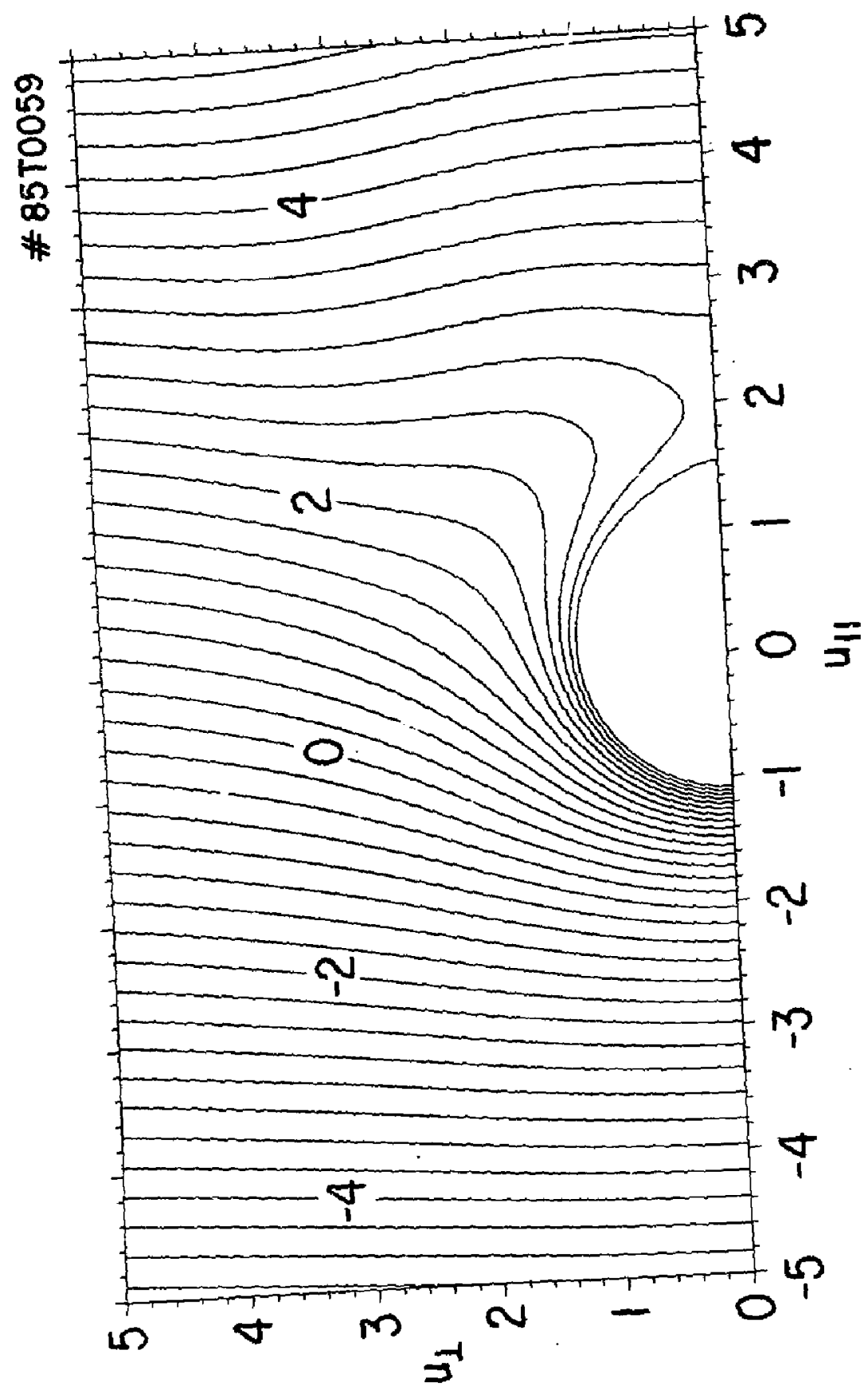

क्षे 


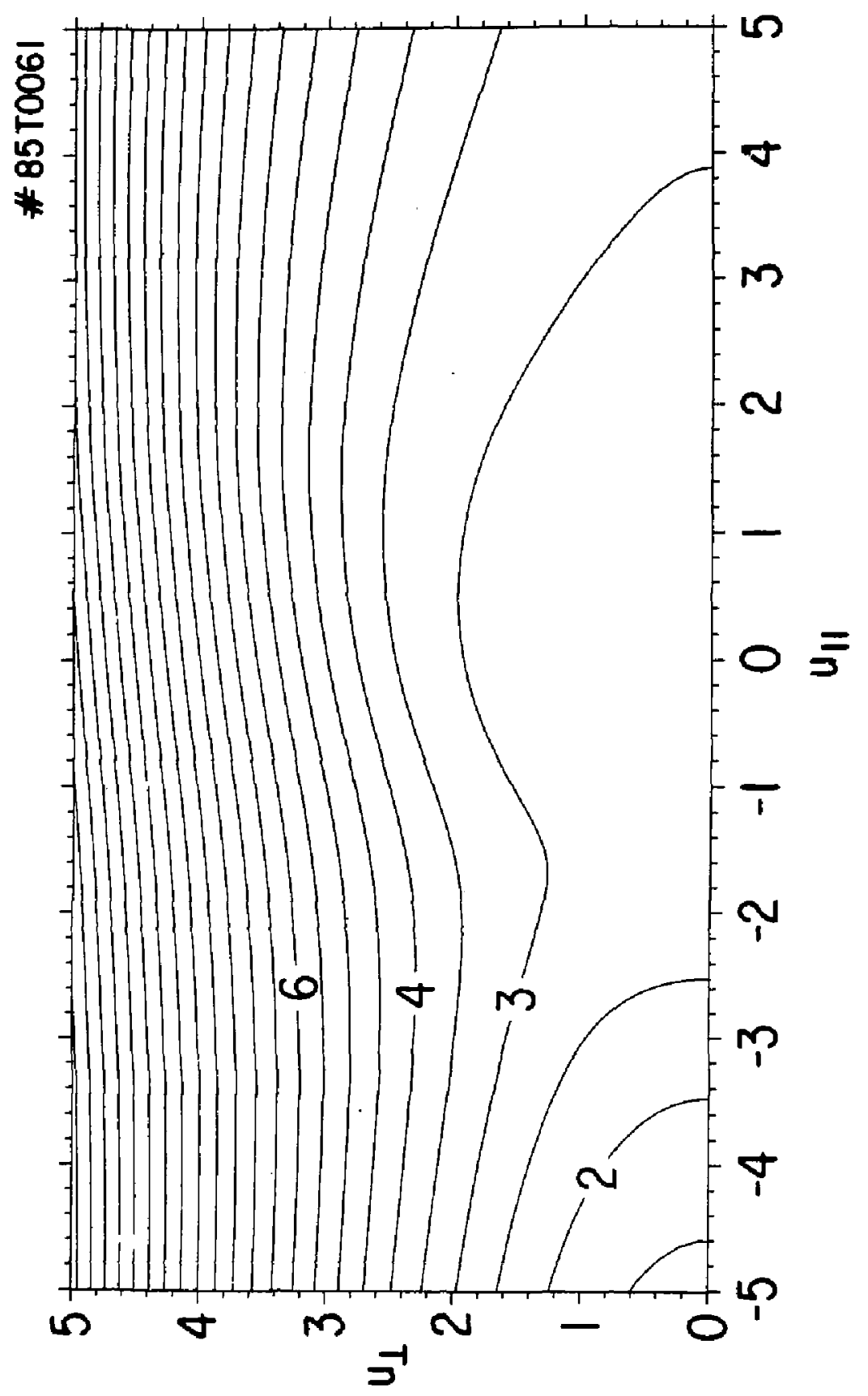

๑ุ 

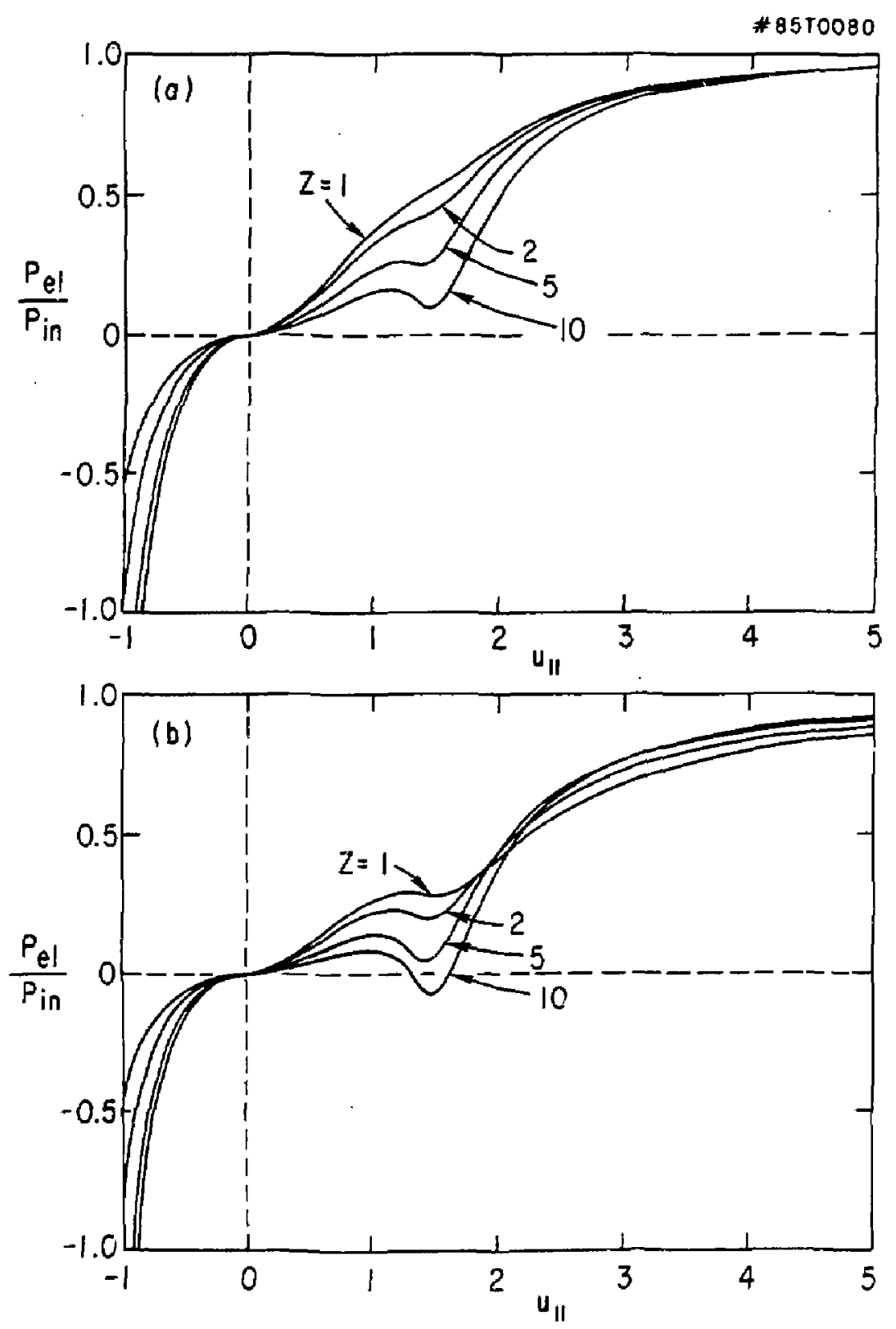

FIg. ? 
Dlaste Res Lab, Austra Nat'1 Univ, AusIRring. Tr. Frants J. Paoloni, Univ of Wollongong, ALSTRALIA Prof. I.R. Janes, Flinders thiv, ALSIRALTA

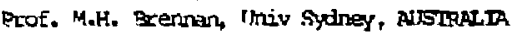
Prof. F. Cap, Inst Iheo Frys, ALSTRIA Prof. Fiark Verheest, Inst theoretlsche, BrignM Dr. D. Palumbo, Dg XII Fusian Prog, esigrum Feole Royali Mlitaire, Lab de Fhys Plasmas, BEIGIM Dr. P.H. Sakanaka, Univ Batakul, BADII, Dr. C.R. Janes, Univ of Alberta, Caraca Prof. J. Teictmara, Univ of itontreal, Carama

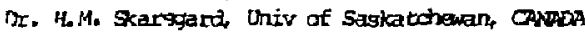
PIof. S. H. Sreenivasan, University of Calgary, CANADA Prof. Tudor W. Joinstor, INRS-brergie, canda Dr. Fannes Bamani, Univ Aritish Coluniaia, Casapa Or. 4.F. Eachynsti, MPB Technologiey, Inc., CANPDA Chalk River, Mucl Lab, CANADA

Thengn Li, SN Ingt Physios, Grom Tibrary, Ising the University, CHND Librarian, Institute of Fhysics, chilta

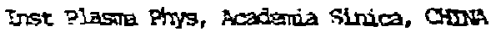
ij. Eeter Lukac, Konengkeho Univ, Cascrostanakds The Lilmarian, Gulhan Laboratroy, Exedan

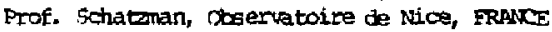
J. Radet, Cal-BPs, FFANE AM Dupas Library, AM Dipas Library, FRAXCE Dr. Iom mal, Acadeny Bibliographic, tants kavg Preprint Library, Cent Res Inst phys, HJNGafy 5r. S.K. Trehan, Panjals Oniversitr, DDIA Dr. Indra kohan Ial Das, Banaras Hindu univ, DDTA Tr. I.K. Chavda, south Gujarat univ, DNIA Or. R.K. Ohaj lani, Vikran thiv. DDIA Jr. A. Dasgnupta, Siha Ingt, DDIA Dr. P. Ka*, Ftrysical Research Lab, InotA Dr. Fhiliip Rosenail, Israel Inst Tech, ISRAEL Prot. S. Duperman, Tel Aviv Univengity, ISRAg. Prof. G. Rostagni, Univ of Pacova, IIALY Libeartan, Int'l Ctr Theo Phys, IDALt

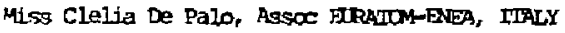
Biblicteca, del ONR BIRATO4, ITPLY

Dr. H. Yamato, Toghtha Res E Dev, JAPAN

Dires. Dept. Lg. Tokamak Dev. JAERT, JAPAN Prot. Nuhnnuki Inave, Untuersity of Rkyo, JAPAN Research Info Center, Nagoya Univergity, JAPAN PCof. Kyoji Nishikana, Unlv of kitroshilma, JAPAN Prof. Sigens Mord, JAERr, IAPis isibrary, Kynto University, JAPAN Prof. IËire Karakami, Nihon Univ, JAPAN Prof. Satwohi Itoh, Kyushu Iniversicy, JAFAN Dr. D.I. Thoi, Hut. Inst Sei \& Tech, KORFA Tech Info Divigion, KAERI, KTREA Fibltothese, Fan-Inst Voor Plagma, NeITERLANDS
Prof. B.S. INley, University of Waikato NES ZEATAND Prof, J.A,C, Cabral, Inst Supertior theon, Porrurat, Dr. Octavian Retus, ALI OZA thiversity, ROUANTA Prof. MA Hellberg, Univensity of Natal, SO AFrICA Dr. Johan de Vllliers, Plasma thysics, Meor, SO AFrreA Fusion Div, Lthary, JoN, SPAII Prof. Hans thilhelmson, Chalmers Univ Tech, SwopN Dr. Lemart Stenflo, University of LMEA, SWrDEN tibrary, Royal Inst Tech, SHDDN

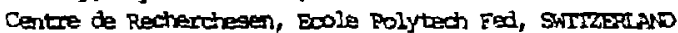
Dr. V.T. Tolok, tharkov Ehys Tech Lns, ISSR Dr. D.D. Fyutow, Siberian Aced Scri, USSR Dx. G.A. Eliseev, Kurchator Instidute, USSR Dr. V.A. Gluthikh, Ingt Electro-Physical, USSR Institute Gen Physica, USSR

Pcof. T.J.M Boyn, Univ College $N$ vales, WaLES Dr. Ko Schindler, Ruhr thiversitat, Muclear Res Pstab, Jultich Lta, w. GPRMaNX Iibrarian, Max-plands Institut, W. GRPMWYY Bibllothek, Inst Plasmaforschmg, W. GFRMAN Prof. R+K. Janev, Inst Phys, Ycoostavia 